\title{
Modélisation macroscopique du transport diffusif Apport des méthodes de changement d'échelle d'espace
}

\author{
L. Dormieux ${ }^{1}$ et E. Lemarchand ${ }^{1}$ \\ 1 CERMMO, ENPC, 6-8, avenue Blaise-Pascal, Champs-sur-Marne, 77455 Marne-la-Vallée Cedex 2 - France \\ e-mail : dormieux@cermmo.enpc.fr, lemarchand@cermmo.enpc.fr
}

\begin{abstract}
Résumé - Cet article propose une modélisation macroscopique du transport diffusif en présence d'advection dans un matériau poreux à partir d'un processus d'homogénéisation. Le domaine de validité est caractérisé par deux paramètres adimensionnels qui permettent de définir respectivement les concepts d'advection modérée et de régime transitoire suffisamment lent.

La méthode d'homogénéisation permet d'estimer les tenseurs de diffusion et de tortuosité introduits dans la formulation macroscopique de la loi de Fick. Ces estimations dépendent des paramètres morphologiques de la microstructure, qui représentent la géométrie du domaine occupé par la phase siège de la diffusion.

Deux techniques différentes d'homogénéisation sont comparées. D'une part, on obtient des estimations en décrivant les grains solides comme des inclusions de la phase fluide. D'autre part, l'homogénéisation périodique est appliquée à des géométries tridimensionnelles de l'espace poreux.

Mots-clés : diffusion, advection, homogénéisation, estimation, périodique, ellipsoïde.
\end{abstract}

\begin{abstract}
Macroscopic Modeling of the Diffusive Transport: Contribution of Upscaling Techniques A modeling at the macroscopic scale for combined advective and diffusive flows in a porous material is presented in this paper as a result of a homogenization process. The domain of validity is characterized by means of two adimensional parameters which respectively allow to define the concepts of moderate advection and slow transient state.

The homogenization method allows to estimate the diffusion and tortuosity tensors introduced in the macroscopic formulation of Fick's law. These estimates depend on the morphological parameters of the microstructure which represent the geometry of the domain occupied by the phase in which the diffusion process takes place.

Two different homogenization techniques are compared. On the one hand, from describing the solid grains as inclusions in the fluid phase inclusion-based estimates are derived. On the other hand, periodic homogenization approach is applied to $3 D$ geometries of the porous space.

Keywords: diffusion, advection, homogenization, estimates, periodic, oblate spheroid.
\end{abstract}




\section{INTRODUCTION}

Le présent travail a pour principal objectif d'établir à l'échelle macroscopique la loi de diffusion d'un soluté dans la phase fluide d'un milieu poreux. Lorsque l'on aborde la description géométrique d'un milieu poreux multiphasique, deux points de vue sont possibles. Le premier consiste à se placer à une échelle d'espace suffisamment fine, dite microscopique dans la suite, pour que les diverses phases constituant un volume élémentaire de milieu poreux apparaissent comme des domaines géométriques distincts. À un instant donné, un point géométrique se trouve donc, à cette échelle, dans une phase bien identifiée. Le second point de vue, dit macroscopique, considère le même volume élémentaire comme la superposition d'autant de particules matérielles qu'il y a de phases, à présent indiscernables géométriquement, c'est-àdire localisées à cette échelle au même point géométrique.

À l'une ou l'autre de ces deux échelles, la description du transport diffusif d'une espèce dans une phase (par exemple du sel dans de l'eau) repose sur le principe de conservation de la masse et sur une modélisation du phénomène de diffusion moléculaire. Partant de l'écriture de ces deux arguments à l'échelle microscopique, un changement d'échelle d'espace permet d'obtenir la formulation homologue à l'échelle macroscopique. Après avoir rappelé le principe du changement d'échelle pour la loi de conservation, on se penchera sur la structure de la modélisation macroscopique du phénomène de diffusion, dont on précisera le domaine de validité à l'aide de paramètres adimensionnels. Il s'agira ensuite d'estimer la valeur des tenseurs de diffusion et de tortuosité que la modélisation macroscopique introduit en fonction des paramètres morphologiques à l'échelle microscopique, qui caractérisent la géométrie de l'espace occupé par la phase siège de la diffusion. À cet effet, on mettra en œuvre, d'une part les méthodes d'estimation des matériaux hétérogènes, et d'autre part les résultats de l'homogénéisation périodique, que l'on appliquera à des descriptions bi- et tridimensionnelles de la géométrie.

\section{CONSERVATION DE LA MASSE}

Partant de l'écriture à l'échelle microscopique d'une loi de conservation, cette section rappelle, sur l'exemple de la conservation de la masse, comment l'on obtient l'écriture du même principe à l'échelle macroscopique. On pourra se reporter par exemple à Gilbert (1987), Bear et Bachmat (1990) pour une application systématique de cette démarche à l'ensemble des lois de conservation. Dans le cadre de ce travail, il s'agit tout particulièrement d'établir la relation entre les descriptions microscopique et macroscopique de la cinématique. Ce point constitue en effet un préalable indispensable dans la perspective de déduire la modélisation macroscopique du phénomène de diffusion de sa modélisation à

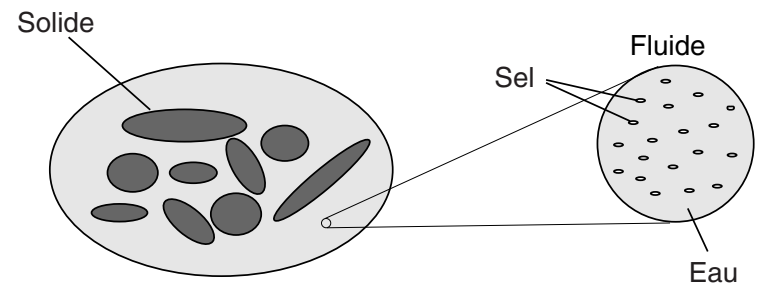

Figure 1

Représentation d'un milieu biphasique : cas d'un fluide à deux composantes.

Representation of a two-phase medium: the case of a fluid with two components.

l'échelle microscopique. Pour simplifier l'exposé, on se place ici dans le cas biphasique (fig. 1).

Les exposants $f$ et $s$ désignent respectivement la phase fluide siège de la diffusion et la phase solide. On note $\rho^{\gamma}$ et $\underline{V}^{\gamma}$ la masse volumique et la vitesse à l'échelle microscopique de l'espèce $\gamma$ de la phase $f$ dans laquelle on étudie la diffusion de cette espèce $\gamma$. L'équation de conservation de la masse à cette échelle, dans le domaine $\boldsymbol{P}^{f}$ occupé par la phase $f$, s'écrit :

$$
\frac{\partial \rho^{\gamma}}{\partial t}+\operatorname{div}_{z} \rho^{\gamma} \underline{V}^{\gamma}=0
$$

où l'opérateur de divergence porte sur la variable d'espace à l'échelle microscopique, notée $\underline{z}$. On note $I^{f s}$ l'interface de la phase fluide avec la phase solide.

Par ailleurs, $\rho_{M}^{\gamma}$ et $\underline{V}_{M}^{\gamma}$ désignant maintenant la masse volumique (intrinsèque) et la vitesse de l'espèce $\gamma$ à l'échelle macroscopique, la loi de conservation macroscopique s'écrit classiquement sous la forme :

$$
\frac{\partial}{\partial t}\left(\varphi^{f} \rho_{M}^{\gamma}\right)+\operatorname{div}_{x} \varphi^{f} \rho_{M}^{\gamma} \underline{V}_{M}^{\gamma}=c(\underline{x}, t)
$$

où la divergence opère à présent sur la variable d'espace macroscopique notée $\underline{x}, \varphi^{f}$ désignant la fraction volumique de la phase $f$ et $c(\underline{x}, t)$ une éventuelle source de masse de l'espèce $\gamma$ à l'échelle macroscopique. La question soulevée est celle de la relation entre le champ $\underline{V}^{\gamma}$ dans un volume élémentaire représentatif et le vecteur $\underline{V}_{M}^{\gamma}$, en sorte que la compatibilité des équations (1) et (2) soit assurée.

\subsection{Dérivations spatiale et temporelle de grandeurs moyennes}

On admet qu'il est possible de définir un volume élémentaire représentatif (v.e.r.) du milieu poreux. Les dimensions caractéristiques des hétérogénéités, du v.e.r. et du système macroscopique étudié, notées respectivement $d, \boldsymbol{R}$ et $\boldsymbol{L}$ sont soumises aux conditions $d<<\boldsymbol{R}<<\boldsymbol{L}$ (fig. 2). 


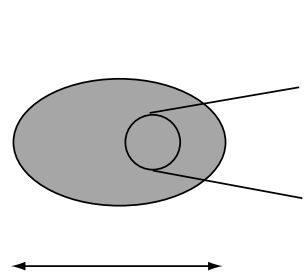

$L$

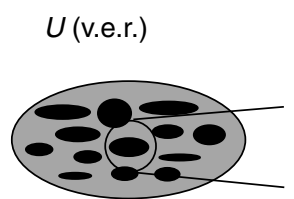

$\boldsymbol{R}$

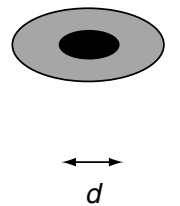

Figure 2

Définition des différentes échelles.

Definition of the different scales.
On introduit tout d'abord l'opérateur de moyenne sur un v.e.r. Plus précisément, soit $U_{o}$ un v.e.r. centré à l'origine de l'espace et $U(\underline{x})=U_{o}+\underline{x}$ le domaine élémentaire obtenu par la translation de vecteur $\underline{x}$ à partir de $U_{o}$. Soit encore $f(\underline{z})$ une fonction infiniment dérivable à support borné dans $U_{o}$, d'intégrale unité. On définit la moyenne $\bar{e}$ au point macroscopique $\underline{x}$ d'une grandeur $e$ par :

$$
\bar{e}(\underline{x}, t)=\int e(\underline{z}, t) f(\underline{z}-\underline{x}) \mathrm{d} V_{z}
$$

Cette définition est évidemment conditionnée par le choix de la fonction $f$. Dans la pratique, $f$ peut être choisie arbitrairement proche de la fonction, $C_{U_{o}} /\left|U_{o}\right|$ où $C_{U_{o}}$ est la fonction caractéristique du domaine $U_{o}$ de volume $\left|U_{o}\right|$. C ${ }^{f}(\underline{z}, t)$ désignant la fonction caractéristique du domaine $\boldsymbol{P} f$, on introduit également les moyennes apparente $\langle\mathrm{e}\rangle_{f}$ et intrinsèque $\bar{e}^{f}$ au point macroscopique $\underline{x}$ d'une grandeur e définie sur $\boldsymbol{P}^{f}$ :

$$
\begin{gathered}
\left\langle e>_{f}(\underline{x}, t)=\overline{C^{f} e}(\underline{x}, t)=\int C^{f}(\underline{z}, t) e(\underline{z}, t) f(\underline{z}-\underline{x}) \mathrm{d} V_{z}\right. \\
\bar{e}^{f}(\underline{x}, t)=\frac{1}{\varphi^{f}}<e>_{f}(\underline{x}, t)
\end{gathered}
$$

$<e>_{f}$ et $\bar{e}^{f}$ sont rapportées respectivement au volume total du v.e.r. et au volume occupé dans le v.e.r. par la phase $f$. Avec ces notations, $\bar{\rho}^{\gamma}$ n'est autre que la masse volumique intrinsèque $\rho_{M}^{\gamma}$ introduite dans l'équation (2).

En vue d'établir l'équation (2) en tant que correspondant macroscopique de l'équation (1), il reste à préciser les règles d'échange des opérations de dérivations spatiale et temporelle avec celle de moyenne. On voit tout d'abord que :

$$
\frac{\partial}{\partial x_{i}}<e>_{f}=-\int C^{f}(\underline{z}, t) e(\underline{z}, t) \frac{\partial f}{\partial z_{i}}(\underline{z}-\underline{x}) \mathrm{d} V_{z}
$$

ou encore, en intégrant par parties au sens de la théorie des distributions :

$$
\frac{\partial}{\partial x_{i}}<e>_{f}(\underline{x}, t)=\int \frac{\partial}{\partial z_{i}}\left(C^{f} e\right)(\underline{z}, t) f(\underline{z}-\underline{x}) \mathrm{d} V_{z}
$$

Notant $\delta_{f s s}$ la distribution de Dirac dont le support est l'interface $I^{f s}$ et $n_{i}$ la $i$-ième composante de la normale unitaire à
$I^{f s}$ orientée de $f$ vers $s$, on montre que $\partial C^{f} / \partial z_{i}=-n_{i} \delta_{f^{f s}}$ Il en résulte que :

$$
\begin{aligned}
\frac{\partial}{\partial x_{i}}<e>_{f}(\underline{x}, t)= & <\frac{\partial e}{\partial z_{i}}(\underline{z}, t)>_{f} \\
& -\int_{I} f_{s} e(\underline{z}, t) n_{i}(\underline{z}, t) f(\underline{z}-\underline{x}) \mathrm{d} S_{z}
\end{aligned}
$$

D'une manière analogue, la dérivation par rapport au temps de $\langle e\rangle_{f}$ fait apparaître un terme supplémentaire à coté de $\langle\partial e / \partial t\rangle_{f}$. En effet, compte tenu de l'équation (4), on obtient :

$$
\begin{aligned}
\frac{\partial}{\partial t}<e>_{f}(\underline{x}, t) & =<\frac{\partial e}{\partial t}(\underline{z}, t)>_{f} \\
& +\int \frac{\partial C^{f}}{\partial t}(\underline{z}, t) e(\underline{z}, t) f(\underline{z}-\underline{x}) \mathrm{d} V_{z}
\end{aligned}
$$

L'intégrale dans l'équation (9) tient compte du mouvement de la frontière de la phase $f$, en l'absence duquel la quantité $\partial C^{f} / \partial t$ est nulle. Plus précisément, en notant $\underline{u}$ la célérité de l'interface $I^{f_{s}}$, on montre que :

$$
\begin{aligned}
\frac{\partial}{\partial t}<e>_{f}(\underline{x}, t) & =<\frac{\partial e}{\partial t}(\underline{z}, t)>_{f} \\
& +\int_{I} f_{s} e(\underline{z}, t)(\underline{u} \cdot \underline{n})(\underline{z}, t) f(\underline{z}-\underline{x}) \mathrm{d} S_{z}
\end{aligned}
$$

\subsection{Formulation macroscopique du principe}

En appliquant les équations (8) avec $e=\rho^{\gamma} V_{i}^{\gamma}$ et (10) avec $e=\rho^{\gamma}$, on obtient par sommation, compte tenu de l'équation (1), la formulation macroscopique du principe de conservation de la masse sous la forme suivante :

$$
\begin{aligned}
\frac{\partial}{\partial t}\left(\varphi^{f} \overline{\rho^{\gamma}} f\right) & +\operatorname{div}_{x}\left(\varphi^{f} \overline{\rho^{\gamma} \underline{V}^{\gamma}} f\right) \\
& =\int_{I^{f s}} \rho^{\gamma}\left(\underline{u}-\underline{V}^{\gamma}\right) \cdot \underline{n} f(\underline{z}-\underline{x}) \mathrm{d} S_{z}
\end{aligned}
$$

La confrontation des équations (11) et (2) fournit d'abord la relation recherchée entre les descriptions de la cinématique aux deux échelles d'espace. La compatibilité des équations (1) et (2) impose en effet que l'on définisse la vitesse macroscopique $\underline{V}_{M}^{\gamma}$ comme la moyenne (intrinsèque) pondérée par les fractions massiques des vitesses dans $\boldsymbol{P}^{f}$ :

$$
\left.\underline{V}_{M}^{\gamma}=\overline{\left(\rho^{\gamma} / \bar{\rho}^{\gamma} f\right.}\right) \underline{V}^{\gamma} f
$$

Par ailleurs, le membre de droite de l'équation (11) permet d'interpréter le terme de source de masse $c$ introduit dans l'équation (2). Il représente le flux de masse de l'espèce $\gamma$ rentrant dans la phase $f$ à travers l'interface $I^{f s}$. Il est nul en l'absence d'échange de l'espèce $\gamma$ entre les phases $f$ et $s$. 


\subsection{Flux de masse advectif, diffusif, dispersif}

On désigne par $\underline{V}=f^{\gamma} \underline{V}^{\gamma}$ la vitesse de la phase définie à l'échelle microscopique dans $\boldsymbol{P}^{f}$ comme la moyenne des vitesses $\underline{V}^{\gamma}$ des diverses espèces de la phase, pondérée par les poids $f^{\gamma}$. On notera que la définition de $\underline{V}$ n'est pas unique, divers choix des $f^{\gamma}$ étant possibles. Il peut s'agir par exemple des fractions volumique, molaire ou encore massique des espèces dans le mélange. On utilisera dans la suite la moyenne intrinsèque $\bar{V}^{f}$ pour représenter la cinématique de la phase à l'échelle macroscopique.

On peut scinder la quantité $\rho^{\gamma} \underline{V}^{\gamma}$ apparaissant dans les équations (1) et (11) en un flux de masse diffusif $j^{\gamma}=\rho^{\gamma}\left(\underline{V}^{\gamma}-\underline{V}\right)$ se rapportant au mouvement relatif de l'espèce $\gamma$ par rapport à celui de la phase $f$, et un flux de masse advectif $\rho^{\gamma} \underline{\underline{V}}$. Pour des raisons qui apparaitront à la section 2.1 , on décompose de plus ce dernier comme la somme de $\bar{\rho}^{\gamma} \underline{V}$ et d'un terme complémentaire $\left(\rho^{\gamma}-\overline{\rho^{\gamma}}\right) \underline{V}$ :

$$
\frac{\partial \rho^{\gamma}}{\partial t}+\operatorname{div}_{z}\left(\bar{\rho}^{\gamma} f \underline{V}+\left(\rho^{\gamma}-\overline{\rho^{\gamma}} f\right) \underline{V}+\underline{j}^{\gamma}\right)=0
$$

On retrouve cette décomposition à l'échelle macroscopique (Bear et Bachmat, 1990) :

$$
{\overline{\rho^{\gamma} \underline{V}^{\gamma}}}^{f}={\overline{\rho^{\gamma}}}^{f} \bar{V}^{f}+\bar{j}^{\gamma} f+{\overline{\tilde{\rho}^{\gamma}}}^{\gamma} \underline{\tilde{V}}^{\gamma}
$$

où $\tilde{a}=a-\bar{a}^{f}$ désigne l'écart de la grandeur $a$ à sa moyenne intrinsèque $\bar{a}^{f}$. Le flux de masse moyen est ainsi décomposé en flux macroscopiques respectivement advectif $\left(\bar{\rho}^{\gamma} \bar{V}^{f}\right)$, diffusif $\left(\bar{j}^{\gamma} f\right)$ et dispersif $\left(\overline{\tilde{\rho}}^{\gamma} f \overline{\tilde{V}}^{f}\right)$, de sorte que l'équation (1) s'écrit encore :

$$
\begin{aligned}
\frac{\partial}{\partial t}\left(\varphi^{f} \bar{\rho}^{\gamma} f\right) & +\operatorname{div}_{x}\left(\varphi^{f} \bar{\rho}^{\gamma} \bar{V}^{f}+<\underline{j}^{\gamma}>_{f}+<\tilde{\rho}^{\gamma} \underline{\tilde{V}}>_{f}\right) \\
& =\int_{I^{f s}} \rho^{\gamma}\left(\underline{u}-\underline{V}^{\gamma}\right) \cdot \underline{n} f(\underline{z}-\underline{x}) \mathrm{d} S_{z}
\end{aligned}
$$

\section{MODÉLISATION MACROSCOPIQUE DU PHÉNOMĖNE DE DIFFUSION}

\subsection{Hypothèses de la modélisation}

La suite de notre propos fait l'hypothèse de la validité de la loi de diffusion de Fick à l'échelle microscopique, où elle s'applique dans tout le volume fluide.
Pour un choix approprié de la vitesse $\underline{V}$ de la phase, on suppose tout d'abord que la diffusion moléculaire de l'espèce $\gamma$ dans la phase $f$ est contrôlée par le gradient de la masse volumique $\rho^{\gamma}$. La loi de Fick prend donc la forme suivante :

$$
\left(\boldsymbol{P}^{\gamma}\right) j^{\gamma}=\rho^{\gamma}\left(\underline{V}^{\gamma}-\underline{V}\right)=-D^{\gamma} \operatorname{grad}_{z} \rho^{\gamma}
$$

où $D^{\gamma}$ est le coefficient de diffusion de $\gamma$ dans un milieu infini rempli de la phase $f$.

Soit à présent $\boldsymbol{V}$ et $\boldsymbol{G}$ les ordres de grandeur de la vitesse $\underline{V}$ et $\mathrm{du}$ gradient de masse volumique microscopique $\operatorname{grad}_{z} \rho^{\gamma}$ dans le système étudié, $\boldsymbol{R}$ et $\boldsymbol{L}$ représentant respectivement comme en section 1.1 la dimension du v.e.r. et celle du système. On admet que le gradient macroscopique $\underline{\operatorname{grad}}_{x} \frac{\overline{\rho^{\gamma}} f}{}$ est également de l'ordre de $\boldsymbol{G}$ (sections 3 et 4.1 ).

Avec ces notations, l'ordre de grandeur du flux advectif $\bar{\rho}^{\gamma} \underline{V}$ dans l'équation (13) est $\boldsymbol{G} \boldsymbol{V} \boldsymbol{L}$, le flux diffusif $\underline{j}^{\gamma}$ étant de l'ordre de $D^{\gamma} \boldsymbol{G}$ et le terme complémentaire $\left(\rho^{\gamma}-\bar{\rho}^{\gamma}\right) \underline{V}$ de l'ordre de $\boldsymbol{G V} \boldsymbol{R}$. On a donc :

$$
\begin{aligned}
& \frac{\left|\left(\rho^{\gamma}-\bar{\rho}^{\gamma} f\right) \underline{V}\right|}{\left|\bar{\rho}^{\gamma} f \underline{V}\right|} \approx \frac{\boldsymbol{R}}{\boldsymbol{L}} \\
& \frac{\left|\left(\rho^{\gamma}-\bar{\rho}^{\gamma} f\right) \underline{V}\right|}{\left|j^{\gamma}\right|} \approx \frac{\boldsymbol{R} \boldsymbol{V}}{D^{\gamma}} \\
& \frac{\mid \bar{\rho}^{\gamma} f}{\left|\underline{j}^{\gamma}\right|} \approx \frac{\boldsymbol{L} \boldsymbol{V}}{D^{\gamma}}
\end{aligned}
$$

Étant donné que $\boldsymbol{R} / \boldsymbol{L}<<1$ (section 1.1), le terme complémentaire est négligeable vis-à-vis des deux autres flux dans l'équation (13) à la condition que $\boldsymbol{R} \boldsymbol{V} / D^{\gamma}<\boldsymbol{R} / \boldsymbol{L}, \bar{\rho}^{\gamma} \underline{V}$ étant alors au plus de l'ordre de $j^{\gamma}$. Ceci correspond à un régime d'advection modérée défini par la restriction $\boldsymbol{V}<D^{\gamma} / \boldsymbol{L}$.

Par ailleurs, soit $t_{c}$ le temps caractéristique des variations de $\rho^{\gamma}$. On pourra négliger $\partial \rho^{\gamma} / \partial t$ dans l'équation (13) au même ordre d'approximation que précédemment si :

$$
\frac{\partial \rho^{\gamma}}{\partial t} /\left|\frac{\partial \underline{j}^{\gamma}}{\partial z_{i}}\right| \approx \frac{\boldsymbol{R}}{\boldsymbol{L}}
$$

Les dérivées spatiales de $\underline{j}^{\gamma}$ étant de l'ordre de $D^{\gamma} \boldsymbol{G} / \boldsymbol{R}$ et la dérivée temporelle de $\rho^{\gamma}$ de l'ordre de $\boldsymbol{L} \boldsymbol{G} / t_{c}$, on voit que l'équation (18) est vérifiée si $t_{c}>\boldsymbol{L}^{2} / D^{\gamma}$. En conclusion, la conservation de la masse d'une espèce $\gamma$ dans une phase $f$ soumise à un régime transitoire suffisamment lent 
$\left(t_{c} \geq \boldsymbol{L}^{2} / D^{\gamma}\right)$ et une advection modérée $\left(\boldsymbol{V} \leq D^{\gamma} / \boldsymbol{L}\right)$ s'exprime donc à l'échelle microscopique par :

$$
\left(\boldsymbol{P}^{f}\right) \operatorname{div}_{z}\left(\underline{j}^{\gamma}+\bar{\rho}^{\gamma} \underline{V}\right)=0
$$

On limitera la suite de cette étude au cas où la phase $f$ est incompressible, ce qui ajoute la condition cinématique $\operatorname{div}_{z} \underline{V}=0$. L'équation (19) se réduit alors à :

$$
\left(\boldsymbol{P}^{f}\right) \operatorname{div}_{z}\left(\underline{j}^{\gamma}\right)=0
$$

On suppose enfin que le flux de masse à travers l'interface $\partial \boldsymbol{P}^{f}$ est nul pour toutes les espèces de la phase $f$ :

$$
\left(\forall \gamma^{\prime}\right) \rho^{\gamma^{\prime}}\left(\underline{V}^{\gamma^{\prime}}-\underline{u}\right) \cdot \underline{n}=0
$$

ce qui se traduit par l'égalité de la vitesse normale de la phase et de la célérité de l'interface $\partial \boldsymbol{P}^{f}$ :

$$
\left(\partial \boldsymbol{P}^{f}\right) \underline{u} \cdot \underline{n}=\underline{V} \cdot \underline{n}
$$

En particulier, pour l'espèce $\gamma$, on déduit des équations (21) et (22) la nullité du flux diffusif normal à $\partial \boldsymbol{P}^{f}$ :

$$
\left(\partial \boldsymbol{P}^{f}\right) \underline{j}^{\gamma} \cdot \underline{n}=0
$$

\subsection{Structure de la modélisation macroscopique}

L'hypothèse d'advection modérée permet de supprimer le terme dispersif $\left.<\tilde{\rho}^{\gamma}{ }^{f} \underline{\tilde{V}}\right\rangle_{f}$ de l'équation (15). En outre, compte tenu de l'équation (21), le membre de droite de cette équation s'annule. La formulation macroscopique du principe de conservation de la masse prend maintenant la forme suivante :

$$
\frac{\partial}{\partial t}\left(\varphi^{f} \bar{\rho}^{f}\right)+\operatorname{div}_{x}\left(\varphi^{f} \bar{\rho}^{\gamma} \underline{V}^{f}+<\underline{j}^{\gamma}>_{f}\right)=0
$$

Considérant la quantité $\underline{\bar{V}}^{f}$ comme une donnée caractérisant l'advection, la résolution du problème de transport de l'espèce $\gamma$ à l'échelle macroscopique passe par une modélisation à cette échelle du flux diffusif macroscopique apparent $<\underline{j}^{\gamma}>_{f}$.

Le flux diffusif $\underline{j}^{\gamma}$ et la masse volumique $\rho^{\gamma}$ sont soumis dans $\boldsymbol{P}^{f}$ à l'équation de champ (20), à la condition aux limites (23) et à la loi de comportement (16). S'inspirant des méthodes utilisées classiquement en mécanique des matériaux hétérogènes, on représente l'existence d'un gradient macroscopique $\underline{H}(\underline{x})=\underline{\operatorname{grad}}_{x} \bar{\rho}^{\gamma} f$ sur le v.e.r. $U(\underline{x})$ par des conditions aux limites écrites sur la partie de la frontière $\partial U(\underline{x})$ du v.e.r. contenue dans la phase $f$ :

$$
\left(\partial U(\underline{x}) \cap \boldsymbol{P}^{f}\right) \rho^{\gamma}(\underline{z})-\rho^{\gamma}(\underline{x})=\underline{H}(\underline{x}) \cdot \underline{z}
$$

L'ensemble des équations (16), (20), (23) et (25) constitue un problème bien posé (à une constante près sur le champ $\left.\rho^{\gamma}\right)$. Bien que l'advection et la variable temps n'y apparaissent pas, il convient de rappeler que les présents développements sont applicables en présence d'advection et en régime transitoire, dans les limites fixées par les inégalités $\boldsymbol{V}<D^{\gamma} / \boldsymbol{L}$ et $t_{c}>\boldsymbol{L}^{2} / D^{\gamma}$.

En vertu de la linéarité du problème (16)-(20)-(23)-(25), dans lequel le gradient macroscopique $\underline{H}$ apparaît comme le paramètre de chargement, le gradient local $\underline{\operatorname{grad}}_{z} \rho^{\gamma}$ s'exprime linéairement en fonction de $\underline{H}$ à l'aide d'un tenseur dit de localisation noté $\underline{\underline{A}}(\underline{z})$ tel que :

$$
\left(\boldsymbol{P}^{f}\right) \underline{\operatorname{grad}}_{z} \rho^{\gamma}(\underline{z})=\underline{\underline{\mathrm{A}}}(\underline{z}) \cdot \underline{H}
$$

Confirmant la modélisation classique du phénomène de diffusion à l'échelle macroscopique, on déduit de l'équation (26), compte tenu de l'équation (16), l'existence d'une relation linéaire entre le flux diffusif macroscopique $\left\langle\underline{j}^{\gamma}\right\rangle_{f}$ et $\underline{H}$ :

$$
<\underline{j}^{\gamma}>_{f}=-\underline{\underline{D}}^{\text {hom }} \cdot \underline{H} \quad \text { avec } \quad \underline{\underline{D}}^{\text {hom }}=\varphi^{f} D^{\gamma} \underline{\underline{A}}^{f}
$$

qui introduit le coefficient de diffusion homogénéisé $\underline{\underline{D}}^{\text {hom }}$, de nature tensorielle. Suivant Bear et Bachmat (1990), on définit le tenseur de tortuosité par la relation suivante :

$$
\underline{T}=\frac{1}{\varphi^{f} D^{\gamma}} \underline{D}^{\text {hom }}
$$

L'apport du processus de changement d'échelle proposé ici réside dans le fait qu'il permet d'interpréter le tenseur $\underline{T}$ de l'équation (28) comme la moyenne intrinsèque du tenseur de localisation :

$$
\underline{\underline{T}}=\underline{\underline{A}}^{f}
$$

Sous cette forme, la tortuosité $\underline{\underline{T}}$ ne dépend manifestement à travers $\underline{\underline{A}}$ que de la géométrie de l'espace poreux. L'intérêt de l'équation (29) est d'ouvrir la voie à la détermination d'estimations des tenseurs de diffusion et de tortuosité en fonction des paramètres caractérisant la morphologie de $\boldsymbol{P}^{f}$ à l'échelle microscopique. C'est l'objet de la section 3 .

\section{ESTIMATIONS DU COEFFICIENT DE DIFFUSION}

Il est fructueux pour les développements qui vont suivre de remplacer le problème réel (16)-(20)-(23)-(25) posé sur $\boldsymbol{P}^{f}$ par un problème fictif posé sur la totalité du v.e.r. $U(\underline{x})$. L'idée consiste en fait à considérer le v.e.r. comme une structure hétérogène du point de vue de ses propriétés diffusives en attribuant un coefficient de diffusion $D^{s}$ au complémentaire $\boldsymbol{P}^{s}$ de $\boldsymbol{P}^{f}$. À cet effet, on introduit un prolongement continu et différentiable de la fonction $\rho^{\gamma}(\underline{z})$ dans $\boldsymbol{P}^{s}$ et un prolongement continu par morceaux de $j^{\gamma}$ dans $\boldsymbol{P}^{s}$. Ce dernier est soumis à la condition de continuité du flux diffusif 
normal $\underline{j} \cdot \underline{n}$ à la traversée d'une éventuelle surface de discontinuité. La loi de conservation (20), les conditions aux limites (25) et la loi de Fick (16) peuvent alors être étendues à tout le v.e.r. :

$$
\begin{gathered}
(U(x)) \operatorname{div}_{z} \underline{j}_{F}^{\gamma}=0 \\
(\partial U(\underline{x})) \rho_{F}^{\gamma}(\underline{z})-\rho_{F}^{\gamma}(\underline{x})=\underline{H}(\underline{x}) \cdot \underline{z} \\
(U(\underline{x})) \underline{j}_{F}^{\gamma}(\underline{z})=-D(\underline{z}) \underline{\operatorname{grad}}_{z} \rho_{F}^{\gamma}
\end{gathered}
$$

avec $D(\underline{z})=D^{\gamma}$ dans $\boldsymbol{P}^{f}$ et $D(\underline{z})=D^{s}$ dans $\boldsymbol{P}^{s}$. L'indice $F$ dans les équations (30), (31) et (32) fait référence au problème fictif. Le passage à la limite $D^{s} \rightarrow 0$ dans les équations (30), (31) et (32) permet de retrouver les équations du problème réel (16)-(20)-(23)-(25). En ce qui concerne la relation (23), on notera qu'elle ne joue plus le rôle de condition aux limites dans le cadre du prolongement à $U(\underline{x})$. Cependant, lorsque $D^{s} \rightarrow 0$, cette équation est une conséquence de la condition de régularité imposée à $\underline{j} \underline{j}^{\gamma}$, prescrivant en particulier la continuité de la composante normale de ce vecteur à la traversée de l'interface $I^{f_{s}}$. En ce sens, le problème fictif (30)-(31)-(32) constitue une généralisation du problème réel. Son intérêt réside dans le fait qu'il est mathématiquement de même nature que les problèmes d'élasticité ou de conductivité thermique dans les matériaux hétérogènes qui ont été largement étudiés dans la littérature et dont il est aisé d'adapter les méthodes (sections 3.1 et 3.2).

Le gradient local de la solution $\rho_{F}^{\gamma}$ du problème fictif étant clairement une fonction linéaire de $\underline{H}$, on généralise l'équation (26) en introduisant un champ de tenseurs de localisation $\underline{A}_{F}(\underline{z})$ défini sur la totalité de $U(\underline{x})$ :

$$
(U(\underline{x})) \underline{\operatorname{grad}}_{z} \rho_{F}^{\gamma}(\underline{z})=\underline{A}_{F}(\underline{z}) \cdot \underline{H}
$$

En vertu de l'équation (31), il est facile de vérifier que le gradient moyen $\underline{\operatorname{grad} \rho_{F}^{\gamma}}$ sur $U(\underline{x})$ n'est autre que $\underline{H}$. Il en résulte que $\overline{\underline{A_{F}}}=\underline{\underline{1}}$, ce qui s'écrit encore :

$$
\varphi^{f}{\overline{\underline{A_{F}}}}^{f}+\left(1-\varphi^{f}\right) \overline{\underline{A}}_{F}^{\mathrm{s}}=\underline{\underline{1}}
$$

Le coefficient de diffusion homogénéisé $\underline{D}_{F}^{\text {hom }}$ du problème fictif est défini par la relation suivante :

$$
\overline{j_{F}^{\gamma}}=-\underline{\underline{D}}_{F}^{\text {hom }} \cdot \underline{H}
$$

En prenant la moyenne de l'équation (33) et en utilisant l'équation (34), on voit que :

$$
\begin{aligned}
\underline{\underline{D}}_{F}^{\text {hom }} & =\varphi^{f} D^{\gamma} \underline{\underline{A}}_{F}^{f}+\left(1-\varphi^{f}\right) D^{s} \overline{\underline{\underline{A}}}_{F}^{s} \\
& =D^{\gamma} \underline{\underline{1}}+\left(1-\varphi^{f}\right)\left(D^{s}-D^{\gamma}\right) \overline{\bar{A}}_{F} s
\end{aligned}
$$

On revient au problème réel à l'aide du passage à la limite $D^{s} \rightarrow 0$ dans l'équation (36), en observant que $\underline{D}_{F}^{\text {hom }}$ tend vers le coefficient de diffusion homogénéisé du problème réel $\underline{D}_{F}^{\text {hom }}$ introduit dans l'équation (27) :

$$
\underline{\underline{D}}^{\text {hom }}=\lim _{D^{s} \rightarrow 0} \underline{D}_{F}^{\text {hom }}=D^{\gamma} \underline{\underline{1}}-\left(1-\varphi^{f}\right) D^{\gamma} \lim _{D^{s} \rightarrow 0} \overline{\underline{A}}_{F}^{s}
$$

Conformément à la définition donnée dans l'équation (28), on déduit de l'équation (37) que :

$$
\underline{\underline{T}}=\frac{1}{\varphi^{\gamma}}\left(\underline{\underline{1}}-\left(1-\varphi^{f}\right) \lim _{D^{s} \rightarrow 0} \overline{\bar{A}}_{F} s\right)
$$

Les estimations du coefficient de diffusion homogénéisé et de la tortuosité sont ainsi ramenées à celle de la quantité ${\overline{\hat{A}_{F}}}^{s}$. En d'autres termes, il s'agit (éq. (33)) d'estimer le gradient moyen dans le domaine $\boldsymbol{P}^{s}$. Afin d'assurer la continuité de la phase fluide, on considère la phase solide comme une phase inclusionnaire vis-à-vis de la phase fluide. Dans cet esprit, nous présentons successivement l'estimation dite de Mori-Tanaka et le résultat du schéma différentiel qui ont vocation à satisfaire cette continuité.

\subsection{Schéma de Mori-Tanaka}

On s'attache dans la suite à rendre compte de la continuité de la phase $f$, siège réel de la diffusion. La phase $s$ est décrite comme une phase inclusionnaire par rapport à la phase $f$.

On considère d'abord la situation extrême où la fraction volumique de $\boldsymbol{P}^{s}$ est suffisamment faible pour que l'on puisse négliger les interactions des particules constituant $\boldsymbol{P}^{s}$. Il est alors possible d'estimer $\overline{\operatorname{grad}}_{z} \rho_{F}^{\gamma}$ en considérant que la particule élémentaire de $\boldsymbol{P}^{s}$, de coefficient de diffusion $D^{s}$, est placée dans un milieu infini de coefficient de diffusion $D^{\gamma}$, avec la condition $\rho_{F}^{\gamma} \rightarrow \underline{H} \cdot \underline{z}$ à l'infini. La solution de ce problème dit de l'inhomogénéité a été donnée par Eshelby (1957) dans le cas où la particule est de forme ellipsoïdale : on montre alors que $\underline{\operatorname{grad}}_{z} \rho_{F}^{\gamma}$ est uniforme dans l'ellipsoïde $\varepsilon$ et donné par :

$$
\left(\underline{\operatorname{grad}}_{z} \rho_{F}^{\gamma}\right)^{\varepsilon}=\left(\underline{\underline{1}}+\left(D^{S}-D^{\gamma}\right) \underline{R}^{\varepsilon}\right)^{-1} \cdot \underline{H}
$$

Le tenseur $\underline{R}_{\gamma}^{\varepsilon}$ intervenant dans l'équation (39) dépend de $D^{\gamma}$ ainsi que de la forme et de l'orientation de l'ellipsoïde $\varepsilon$. Plus précisément, on introduit le potentiel $\varnothing(\underline{z})$ de $\varepsilon$ défini par:

$$
\emptyset(\underline{z})=\int_{\varepsilon} \frac{\mathrm{d} V_{Z}}{|\underline{z}-\underline{Z}|}
$$


La restriction à $\varepsilon$ de $\varnothing(\underline{z})$ est un polynôme du second degré en $z_{i}$. Dans un repère orthonormé dont les axes coïncident avec ceux de l'ellipsoïde, $\varnothing(\underline{z})$ est de la forme :

$$
\emptyset(\underline{z})=\frac{1}{2}\left(I_{1}\left(a_{1}-z_{1}\right)^{2}+I_{2}\left(a_{2}-z_{2}\right)^{2}+I_{3}\left(a_{3}-z_{3}\right)^{2}\right)
$$

où les $a_{i}$ sont les longueurs des demi-axes de l'ellipsoïde et les $I_{i}$ sont des coefficients constants fonction des $a_{i}$. On montre (annexe 1) que les composantes du tenseur $\underline{R}_{\gamma}^{\varepsilon}$ sont données par :

$$
\stackrel{R}{=}_{\gamma i j}^{\varepsilon}=-\frac{1}{4 \pi D^{\gamma}} \phi_{, i j}
$$

ce qui implique que ces quantités sont des constantes dans $\varepsilon$. L'uniformité de $\underline{\operatorname{grad}}{ }_{z} \rho_{F}^{\gamma}$ dans l'ellipsoïde en résulte. On verra par la suite qu'il est commode d'introduire le tenseur d'Eshelby $\underline{S}^{\text {Esh }}$, qui est indépendant du coefficient de diffusion $D^{\gamma}$ :

$$
S_{i j}^{\text {Esh }}=\underline{R}_{=\gamma i j}^{\varepsilon} D^{\gamma}=-\frac{1}{4 \pi} \phi_{, i j}
$$

Le coefficient de diffusion homogénéisé du problème fictif, dans le cas dilué, est déduit des équations (36) et (39) :

$$
\underline{D}_{F}^{D}=D^{\gamma} \underline{\underline{1}}+\left(1-\varphi^{f}\right)\left(D^{s}-D^{\gamma}\right)\left(\underline{\underline{1}}+\left(D^{s}-D^{\gamma}\right) \underline{\underline{R}}_{\gamma}^{\varepsilon}\right)^{-1}
$$

Le passage à la limite $D^{s} \rightarrow 0$ donne donc, d'après les équations (37), (38) et (43) :

$$
\left\{\begin{array}{l}
\underline{\underline{D}}^{D}=D^{\gamma}\left(\underline{\underline{1}}-\left(1-\varphi^{f}\right)\left(\underline{\underline{1}}-\underline{\underline{S}}^{\mathrm{Esh}}\right)^{-1}\right) \\
\underline{\underline{T}}^{D}=\frac{1}{\varphi^{f}}\left(\underline{\underline{1}}-\left(1-\varphi^{f}\right)\left(\underline{\underline{1}}-\underline{\underline{S}}^{\mathrm{Esh}}\right)^{-1}\right)
\end{array}\right.
$$

Rappelons que la validité de cette estimation repose sur l'hypothèse que la fraction volumique de $\boldsymbol{P}^{s}$ est infinitésimale. Elle porte à ce titre le nom d'estimation diluée. Pour dépasser cette restriction, il convient de gérer l'interaction entre les particules de $\boldsymbol{P}$. À cet effet, le schéma classique (Zaoui, 1996) introduit une adaptation de la démarche précédente. Il propose d'estimer $\overline{\operatorname{grad}}_{z} \rho_{F}^{\gamma}$ par la valeur du gradient (uniforme) qui s'établit dans une inclusion ellipsoïdale de forme et d'orientation bien choisies, placée comme précédemment dans un milieu infini de coefficient de diffusion $D^{\gamma}$, mais où la condition à l'infini est à présent $\rho_{F}^{\gamma} \rightarrow \underline{H}_{f} \cdot \underline{z}, \underline{H}_{f}$ étant le gradient moyen dans la phase $f$. On a donc :

$$
\underline{\operatorname{grad}}_{z} \rho_{F}^{\gamma} s=\left(\underline{\underline{1}}+\left(D^{s}-D^{\gamma}\right) \underline{\underline{R}}_{\gamma}^{\varepsilon}\right)^{-1} \cdot \underline{H}_{f}
$$

Puis, la relation (34) permet d'éliminer $\underline{H}_{f}$ au profit de $\underline{H}$ :

$$
\underline{H}_{f}=\left(\varphi^{f} \underline{\underline{1}}+\left(1-\varphi^{f}\right)\left(\underline{\underline{1}}+\left(D^{S}-D^{\gamma}\right) \underline{R}^{\varepsilon}\right)^{-1}\right)^{-1} \cdot \underline{H}
$$

En reportant ce résultat dans l'équation (46), on trouve donc, compte tenu de l'équation (33) :

$$
\begin{aligned}
\overline{\underline{A}}_{F}^{s}= & \left(\underline{\underline{1}}+\left(D^{s}-D^{\gamma}\right) \underline{\underline{R}}_{\gamma}^{\varepsilon}\right)^{-1} \cdot \mid \\
& \left(\varphi^{f} \underline{\underline{1}}+\left(1-\varphi^{f}\right)\left(\underline{\underline{1}}+\left(D^{s}-D^{\gamma}\right) \underline{\underline{R}}_{\gamma}^{\varepsilon}\right)^{-1}\right)^{-1}
\end{aligned}
$$

Compte tenu de l'équation (38), l'estimation de $\underline{\underline{T}}$ correspondant à l'équation (48) est :

$$
\begin{aligned}
\underline{T}^{\mathrm{MT}}= & \frac{1}{\varphi^{f}}\left(\underline{\underline{1}}-\left(1-\varphi^{f}\right)\left(\underline{\underline{1}}-\underline{\underline{S}}^{\mathrm{Esh}}\right)^{-1}\right. \\
& \left.\cdot\left(\varphi^{f} \underline{\underline{1}}+\left(1-\varphi^{f}\right)\left(1-\underline{\underline{S}}^{\mathrm{Esh}}\right)^{-1}\right)^{-1}\right)
\end{aligned}
$$

Examinons le cas particulier où la phase $s$ est représentée par des inclusions de forme sphérique. La restriction à la sphère de rayon $a$ du potentiel $\emptyset(\underline{z})$ de l'équation (40) ne dépend que de la distance $r$ du point $\underline{z}$ au centre de la sphère, et l'équation (41) se simplifie :

$$
\varnothing(\underline{z})=\frac{2}{3} \pi\left(3 a^{2}-r^{2}\right)
$$

Il résulte des équations (42), (43) et (50) que $\underline{\underline{R}}_{\gamma}^{\varepsilon}$ et $\underline{S}^{\text {Esh }}$ sont des tenseurs sphériques :

$$
\stackrel{R}{=}_{\gamma}^{\varepsilon}=\frac{1}{3 D^{\gamma}} \stackrel{1}{=} \text { et } \underline{\underline{S}}^{\mathrm{Esh}}=\frac{1}{3} 1
$$

En utilisant l'équation (51) dans l'équation (49), on obtient les expressions suivantes du coefficient de diffusion homogénéisé et de la tortuosité, conformes aux résultats classiques de Maxwell (1892) qui, le premier, a obtenu des expressions similaires dans la détermination de la conductivité électrique de matériaux hétérogènes ; ces expressions sont, en outre, analogues à celles obtenues par Hashin (1968) sur l'exemple de l'équation de la chaleur :

$$
\underline{\underline{D}}_{\mathrm{sph}}^{\mathrm{MT}}=\frac{2 \varphi^{f}}{3-\varphi^{f}} D^{\gamma} \underline{\underline{1}} ; \quad \underline{T}_{\mathrm{sph}}^{\mathrm{MT}}=\frac{2}{3-\varphi^{f}} \stackrel{1}{=}
$$

La variation de $\underline{\underline{D}}_{\mathrm{sph}}^{\mathrm{MT}}$ en fonction de la porosité est représentée en figure 3. Les estimations données ci-dessus ont évidemment l'attrait de la simplicité. De plus, pour toute morphologie satisfaisant l' hypothèse de répartition isotrope des phases $f$ et $s, \underline{T}_{\mathrm{sph}}^{\mathrm{MT}}$ possède le statut de majorant de la valeur exacte de la tortuosité, au sens mathématique suivant :

$$
(\forall \underline{X},|\underline{X}|=1) \underline{X} \cdot \underline{T} \cdot \underline{X} \leq T_{\mathrm{sph}}^{\mathrm{MT}}=\frac{2}{3-\varphi^{f}}
$$




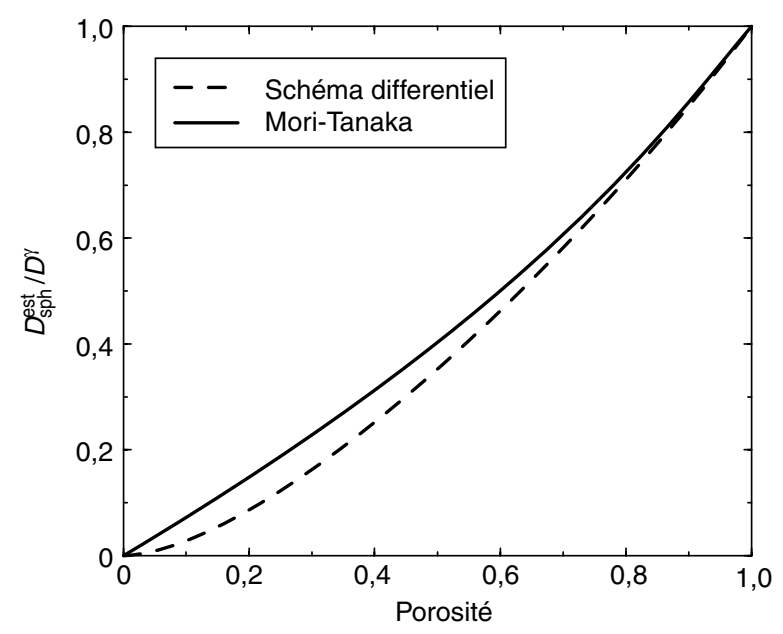

Figure 3

Estimations du coefficient de diffusion : le cas sphérique.

Diffusion coefficient estimates: the case of spherical inclusions.

L'équation (53) est la transposition d'un résultat classique dû à Hashin et Shtrikman (1963) au problème de la détermination du tenseur $\underline{\underline{D}}^{\text {hom }}$ dans l'équation (27).

On vérifie en utilisant l'équation (51) dans l'équation (45) que les estimations diluées $\underline{D}_{\mathrm{sph}}^{D}$ et $\stackrel{T}{=}_{\mathrm{sph}}^{D}$ ne sont autres que les développements limités au voisinage de $\varphi^{f}=1$ de $\underline{\underline{D}}_{\mathrm{sph}}^{\mathrm{MT}}$ et $\underline{T}_{\text {sph }}^{\mathrm{MT}}$ :

$$
\begin{aligned}
& \underline{D}_{\mathrm{sph}}^{D}=\frac{1}{2}\left(3 \varphi^{f}-1\right) D^{\gamma} \underline{\underline{1}} \\
& \text { et } \quad \stackrel{T}{=}_{\mathrm{sph}}^{D}=\frac{1+\varphi^{f}}{2} \underline{1}
\end{aligned}
$$

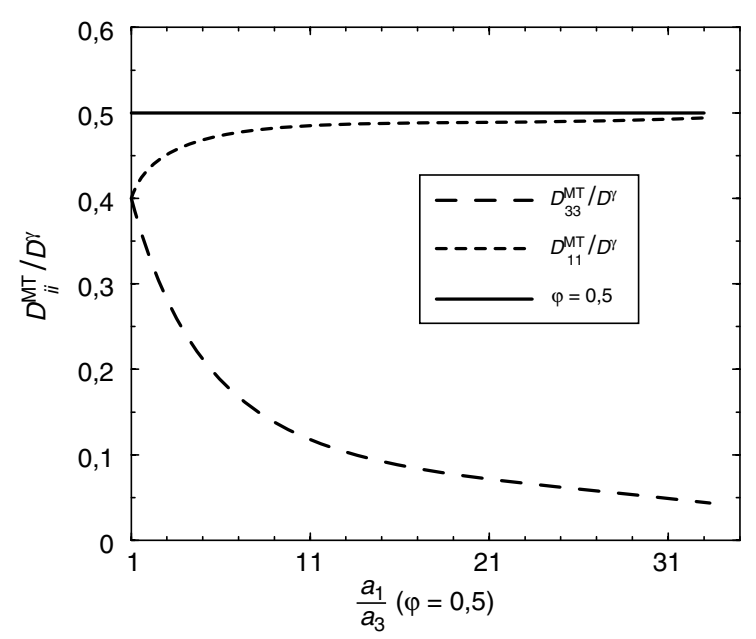

Figure 4

Influence de l'anisotropie (Mori-Tanaka).

Influence of anisotropy (Mori-Tanaka).
Les estimations de Mori-Tanaka dans le cas non sphérique sont évidemment plus complexes, bien que demeurant analytiques. Elles sont données en annexe 1. Notons qu'un résultat dû à Willis (1977) généralise le statut de borne (majorant) de l'estimation de Mori-Tanaka vis-à-vis de la valeur exacte du tenseur $\underline{D}^{\text {hom }}$ pour le cas d'inclusions ellipsoïdales de mêmes forme et orientation, dans l'hypothèse d'une répartition ellipsoïdale des phases. Il résulte des équations (41), (42) et (49) que les directions principales de $\underline{\underline{D}}^{\mathrm{MT}}$ et $\underline{\underline{T}}{ }^{\mathrm{MT}}$ coïncident avec les axes de l'ellipsoïde $\varepsilon$. L'orientation et la forme de ce dernier contrôlent l'anisotropie de ces tenseurs. À titre d'exemple, on s'intéresse au cas où l'ellipsoïde $\varepsilon$ posséde la symétrie de révolution autour de l'axe $\underline{e}_{3}$ d'un repère orthonormé $\left(\underline{e}_{1}, \underline{e}_{2}, \underline{e}_{3}\right)$, et l'on examine l'effet sur l'anisotropie du tenseur de diffusion d'un aplatissement de l'ellipsoïde. Désignant par $a_{i}$ la demi-longueur de l'axe de $\varepsilon$ de vecteur $\underline{e}_{i}$ (avec $a_{1}=a_{2}$ ), les figures 4 et 5 représentent les estimations des coefficients $\underline{D}_{11}^{\mathrm{MT}}$ et $\underline{\underline{D}}_{33}^{\mathrm{MT}}$ du tenseur de diffusion respectivement fonction des rapports d'axe pour $\varphi=0,5$ et fonction de la porosité pour $a_{1} / a_{3}=3$. Comme prévu, l'anisotropie du phénomène de diffusion mesurée par le rapport $\underline{D}_{11}^{\mathrm{MT}} / \underline{\underline{D}}_{33}^{\mathrm{MT}}$ croît avec le rapport $a_{1} / a_{3}$. Pour un aplatissement croissant, on note également que le coefficient $\underline{D}_{11}^{\mathrm{MT}}$ qui représente le coefficient de diffusion pour un gradient macroscopique orienté parallèlement au plan $\underline{e}_{1}, \underline{e}_{2}$ (grands axes de l'ellipsoïde) tend vers une variation proportionnelle à la porosité (loi des mélanges). Intuitivement, cela signifie que l'aplatissement des particules solides autorise un mouvement quasi rectiligne des molécules parallèlement à la direction du gradient macroscopique, et supprime la tortuosité de leurs trajectoires. Au contraire, pour un gradient macroscopique colinéaire à $\underline{e}_{3}$, l'aplatissement fait croître la tortuosité. Ainsi, à porosité fixée, $\underline{D}_{11}^{\mathrm{MT}}$ (respectivement

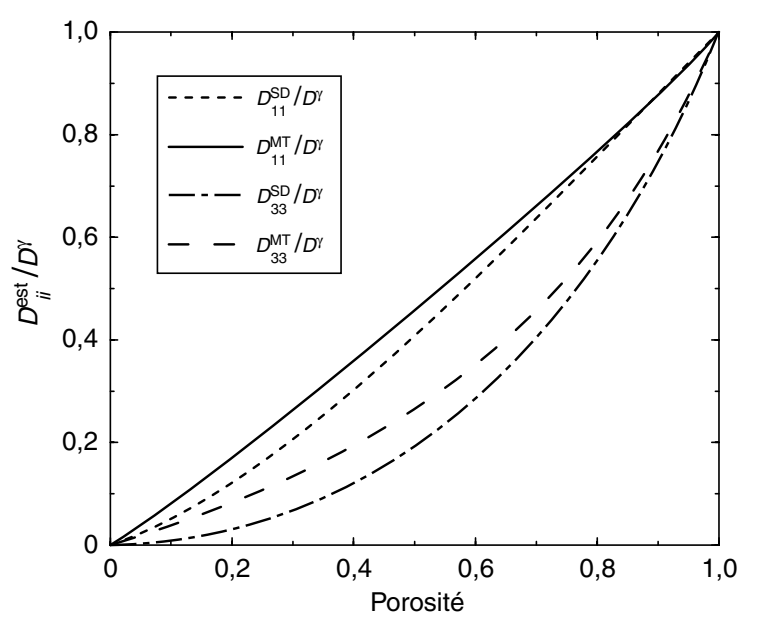

Figure 5

Anisotropie du tenseur de diffusion : $a_{1} / a_{3}=3$.

Diffusion tensor anisotropy: $a_{1} / a_{3}=3$. 
$\underline{\underline{D}}_{33}^{\mathrm{MT}}$ ) croît (respectivement décroît) avec le rapport $a_{1} / a_{3}$. Ces considérations sont illustrées par le schéma de principe de la figure 6. Elles s'appuient sur l'interprétation géométrique du coefficient de tortuosité proposée à la section 4.3. À cet égard, on prendra garde au fait que le coefficient de tortuosité est d'autant plus grand (proche de 1) que le trajet est moins « tortueux ».
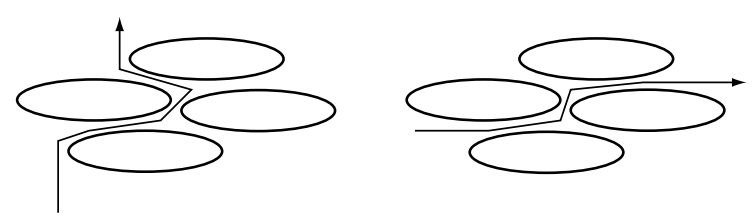

Figure 6

Influence de l'aplatissement sur l'anisotropie du tenseur de tortuosité.

Influence of inclusion aspect ratio on the anisotropy of the tortuosity tensor.

\subsection{Schéma différentiel}

À côté du schéma de Mori-Tanaka, le schéma différentiel constitue une autre façon, plus progressive mais aussi plus complexe, d'introduire le caractère continu de la phase siège de la diffusion dans la démarche d'estimation de la tortuosité (Zaoui, 1997). Comme celui de Mori-Tanaka, il se présente sous la forme d'une adaptation de l'estimation diluée. L'idée consiste à introduire la phase $s$ (inclusionnaire) dans la phase $f$ (matrice) par incréments infinitésimaux successifs à partir d'un milieu homogène de coefficient de diffusion $D^{\gamma}$, jusqu'à ce que la valeur désirée de la fraction volumique de $\boldsymbol{P}^{s}$ soit atteinte. Après chaque incrément, on détermine le milieu homogène équivalent qui est utilisé pour l'itération suivante. La première itération est celle conduisant à l'estimation diluée (45). Soit $\underline{\underline{D}}_{F}^{k}$, le coefficient de diffusion homogénéisé réalisé à la $k$-ième itération. On a donc $\underline{\underline{D}}_{F}^{0}=D^{\gamma_{1}}$.

La $k+1$-ième itération consiste à enlever une fraction infinitésimale $\mathrm{d} \varepsilon<1 \mathrm{du} k$-ième milieu homogène et à la remplacer par le même volume de la phase $s$. Il en résulte une variation $\mathrm{d} \varphi^{s}=-\mathrm{d} \varphi^{f}$ égale à $\varphi_{k}^{f} \mathrm{~d} \varepsilon$. Comme $\mathrm{d} \varepsilon$ est infinitésimal, le $k+1$-ième milieu homogénéisé peut être déterminé par l'estimation diluée introduite à la section 3.1. En remplaçant dans l'équation (44) $\mathrm{D}^{\gamma} \underline{\underline{1}}$ par $\underline{\underline{D}}_{F}^{k}$ et $\left(1-\varphi^{f}\right)$ par $\mathrm{d} \varepsilon$, on obtient une relation de récurrence conduisant à l'estimation du tenseur de diffusion homogénéisé $\underline{D}_{F}^{\text {hom }}$ de l'équation (35) :

$$
\begin{aligned}
\underline{\underline{D}}_{F}^{k+1}= & \underline{\underline{D}}_{F}^{k}+\mathrm{d} \varepsilon\left(D^{s} \underline{\underline{1}}-\underline{\underline{D}}_{F}^{k}\right) \\
& \cdot\left(\underline{\underline{1}}+\left(D^{s} \underline{\underline{1}}-\underline{\underline{D}}_{F}^{k}\right) \cdot \underline{\underline{R}}_{F}^{\varepsilon k}\right)^{-1}
\end{aligned}
$$

Le tenseur $\underline{R}_{F}^{\varepsilon k}$ intervenant dans l'équation (55) dépend comme précédemment de la forme et de l'orientation de l'ellipsoïde $\varepsilon$ mais aussi du coefficient de diffusion $\underline{\underline{D}}_{F}^{k}$ définissant le $k$-ième milieu homogénéisé. La relation (42), valable pour le premier incrément, donne la valeur de $\underline{\underline{R}}_{F}^{\varepsilon 0}$. Le calcul de $\underline{R}_{F}^{\varepsilon k}$ pour $k \geq 1$ est détaillé en annexe 1 . Compte tenu de l'équation (55), les directions principales des tenseurs $\underline{\underline{D}}_{F}^{k}$ coïncident avec les axes de l'ellipsoïde.

Un passage à la limite $D^{s} \rightarrow 0$ dans l'équation (55) fournit la relation de récurrence conduisant à l'estimation du tenseur de diffusion homogénéisé $\underline{\underline{D}}^{\text {hom }}$ de l'équation (27) :

$$
\underline{\underline{D}}^{k+1}=\underline{\underline{D}}^{k}-\mathrm{d} \varepsilon \underline{\underline{D}}^{k} \cdot\left(\underline{\underline{1}}_{-}-\underline{\underline{S}}^{\mathrm{Esh}}{ }^{k}\right)^{-1}
$$

La forme différentielle de l'équation (56) s'écrit :

$$
\mathrm{d} \underline{\underline{D}}=\frac{\mathrm{d} \varphi^{f}}{\varphi^{f}} \underline{\underline{D}} \cdot\left(\underline{\underline{1}}_{-}-\underline{\underline{S}}^{\text {Esh }}\right)^{-1}
$$

Le tenseur $\underline{\underline{D}}^{\text {hom }}$ est obtenu par intégration de l'équation (57) par rapport à $\varphi^{f}$ entre 1 et la valeur désirée.

Dans le cas particulier où la phase $s$ est représentée par des inclusions de forme sphérique, le schéma différentiel peut être mis en œuvre analytiquement. En effet, le milieu homogénéisé restant isotrope après chaque incrément, le tenseur $\underline{\underline{D}}^{k}$ est de la forme $D^{k} \underline{\underline{1}}$. Le tenseur $\underline{R}^{\varepsilon k}$, obtenu en remplaçant $D^{\gamma}$ par $D^{k}$ dans l'équation (42), est donc égal à $1 /\left(3 D^{k}\right) \underline{\underline{1}}$, de sorte que l'équation (57) devient :

$$
\frac{\mathrm{d} D}{D}=\frac{3 \mathrm{~d} \varphi^{f}}{2 \varphi^{f}}
$$

dont l'intégration donne :

$$
\underline{\underline{D}}^{S D}=D^{\gamma}\left(\varphi^{f}\right)^{3 / 2} \underline{\underline{1}} ; \underline{\underline{T}}^{S D}=\left(\varphi^{f}\right)^{1 / 2} \underline{\underline{1}}
$$

La variation de $\underline{\underline{D}}_{\mathrm{sph}}^{S D}$ en fonction de la porosité est représentée à la figure 3, qui fait apparaître que le coefficient de diffusion prévu par le schéma différentiel est légèrement inférieur à l'estimation de Mori-Tanaka.

Dans le cas de l'inclusion non sphérique, l'intégration de l'équation (57) doit être effectuée numériquement. Les résultats sont présentés en figure 5. En comparaison avec l'estimation de Mori-Tanaka, on note que le schéma différentiel accentue le rapport d'anisotropie $D_{11} / D_{33}$.

\section{APPROCHE DE LA DIFFUSION MACROSCOPIQUE PAR L'HOMOGÉNÉISATION PÉRIODIQUE}

De façon alternative par rapport à l'approche développée dans les sections 2 et 3 , une modélisation macroscopique du phénomène de diffusion peut être construite à partir d'un changement d'échelle, sur la base d'une hypothèse de 
morphologie périodique du réseau poreux. Après en avoir rappelé le principe, on met en œuvre numériquement cette approche sur diverses morphologies. Les résultats obtenus sont confrontés à ceux de la section 3 .

\subsection{Principe de l'homogénéisation périodique}

On se limite ici à donner d'une manière simplifiée l'idée de la méthode périodique en l'appliquant au problème défini par les équations (16), (20) et (23). Les conclusions de cette approche sont conformes aux résultats de Auriault et Lewandowska (1996). On trouvera de plus chez ces auteurs une démarche systématique permettant notamment de statuer (dans le cadre périodique) lorsque l'advection ou les variations temporelles ne sont pas compatibles avec les hypothèses de la section 2.1 .

Le réseau poreux est obtenu en reproduisant de manière périodique une cellule de base $\boldsymbol{C}$ dont la géométrie est entièrement définie. On note $\boldsymbol{C}^{f}$ le sous-domaine de $\boldsymbol{C}$ occupé par la phase fluide. Pour simplifier l'exposé, on suppose que $\boldsymbol{C}$ est un parallélépipède dont les arètes, de longueur $l_{i}$, sont parallèles aux vecteurs $\underline{e}_{i}$ d'une base orthonormée. Il est délimité par les plans $z_{i}=0$ et $z_{i}=l_{i}$. $L$ désignant comme précédemment la dimension caractéristique du système, le rapport $\varepsilon=\sup _{i} l_{i} / L$ est infiniment petit.

Pour prendre en compte la morphologie périodique, on impose des propriétés de périodicité à la concentration $\rho^{\gamma}$ et au flux diffusif $j^{\gamma}$. Ainsi, on recherche la fonction $\rho^{\gamma}$ sur une cellule élémentaire sous la forme de la somme d'un terme affine $\rho_{0}^{\gamma}+\underline{H} \cdot \underline{z}$ rendant compte des variations de $\rho^{\gamma}$ à l'échelle macroscopique et d'une fluctuation périodique $r(\underline{z})$ :

$$
\rho^{\gamma}(\underline{z})=\rho_{0}^{\gamma}+\underline{H} \cdot \underline{z}+r(\underline{z})
$$

la périodicité de la fonction $r(\underline{z})$ s'écrivant :

$$
\left(\forall \underline{z} \in I^{f s} \cap\left(z_{i}=0\right)\right) r\left(\underline{z}+l_{i} \underline{e}_{i}\right)=r(\underline{z})
$$

De même, compte tenu des équations (16) et (60), on décompose le flux diffusif sous la forme :

$$
\underline{j}^{\gamma}=-D^{\gamma} \underline{H}+\underline{f}
$$

où $\underline{f}=-D^{\gamma} \underline{\operatorname{grad}}_{z} r$ possède la propriété de périodicité suivante :

$$
\left(\forall \underline{z} \in \partial C^{f} \cap\left(z_{i}=0\right)\right) f_{i}\left(\underline{z}+l_{i} \underline{e}_{i}\right)=f_{i}(\underline{z})
$$

Désignant par $\underline{n}$ la normale à la frontière de la cellule, l'équation (63) exprime la périodicité de la composante normale $(\underline{f} \cdot \underline{n}) \underline{n}$ du flux $\underline{f}$, et donc du gradient $\underline{\operatorname{grad}} r \mathrm{r}$ de $r(\underline{z})$. Comme l'équation (25), les équations (60), (61) et (63) visent à conférer à $\underline{H}$ le sens physique de gradient macroscopique de masse volumique. En introduisant la forme (60) de $\rho^{\gamma}$ dans l'équation (16), les équations (20) et (23) conduisent à un problème différentiel sur la fluctuation $r(\underline{z})$ :

$$
\begin{gathered}
\left(\boldsymbol{C}^{f}\right) \Delta_{z} r=0 \\
\left(I^{f s}\right) \underline{\operatorname{grad}}_{z} r \cdot \underline{n}=-\underline{H} \cdot \underline{n}
\end{gathered}
$$

Compte tenu des conditions de périodicité (61) et (63), les équations (64) et (65) déterminent la valeur de la fluctuation $r(\underline{z})$ à une constante près, que l'on peut fixer en imposant que la moyenne $\bar{r}^{f}$ de $r$ sur $C^{f}$ soit nulle. Avec cette convention, il est clair que $r(\underline{z})$ dépend linéairement de $\underline{H}$. Il existe donc un champ $\chi(\underline{z})$, fonction seulement de la morphologie de $\boldsymbol{C}^{f}$, tel que :

$$
r(\underline{z})=\underline{\chi}(\underline{z}) \cdot \underline{H}
$$

On déduit alors des équations (60) et (66) la valeur en fonction de $\underline{\chi}$ du tenseur de localisation $\underline{A}_{\text {per }}$, homologue dans le cas périodique à celui introduit dans l'équation (26) :

$$
\begin{array}{r}
\left(C^{f}\right) \underline{\operatorname{grad}} \underline{z}_{z}^{\gamma}=\underline{A}_{\text {per }}(\underline{z}) \cdot \underline{H} \\
\text { avec } \underline{A}_{\text {per }}=\underline{\underline{1}}+{ }^{t} \underline{\underline{\operatorname{grad}}}_{z} \underline{\chi}
\end{array}
$$

Comme dans l'équation (27), le calcul du flux diffusif macroscopique $\left\langle\underline{j}^{\gamma}\right\rangle_{f}$ intervenant dans l'équation (24) en fonction du gradient macroscopique $\underline{H}$ amène à introduire un coefficient de diffusion homogénéisé et un tenseur de tortuosité :

$$
\begin{aligned}
<\underline{j}^{\gamma}>_{f} & =-\underline{D}_{\mathrm{per}}^{\mathrm{hom}} \cdot \underline{H} \\
\text { avec } \underline{D}_{\mathrm{per}}^{\text {hom }} & =\varphi^{f} D^{\gamma} \underline{T} \underline{\mathrm{T} e r}
\end{aligned}
$$

Comme dans l'équation (29), le tenseur de tortuosité $\underline{T}=$ per propre au modèle périodique apparaît comme la moyenne intrinsèque du tenseur de localisation :

$$
\underline{T}_{\text {per }}=\underline{\bar{A}}_{\text {per }}^{f}=\underline{\underline{1}}+{ }^{t \overline{\operatorname{grad}}_{z}} \underline{\underline{\underline{\chi}}}
$$

On voit que la détermination du tenseur de tortuosité à partir de la connaissance de la morphologie de $\boldsymbol{C}^{f}$ passe par le calcul de la solution périodique (de moyenne nulle) du problème (64)-(65). Dans la perspective de la résolution numérique, il convient d'aborder cette question du point de vue variationnel.

\subsection{Approche variationnelle de I'homogénéisation périodique}

L'approche variationnelle consiste à caractériser la composante $\underline{f}=-D^{\gamma} \underline{\operatorname{grad}}_{z} r$ du flux diffusif $\underline{j}^{\gamma}$ et la fluctuation $r(\underline{z})$, solutions du problème direct (61)-(63)-(64)-(65), par le fait qu'elles réalisent le minimum d'une fonctionnelle. 
On définit d'abord l'ensemble $\boldsymbol{F}$ des flux admissibles $f$ définis de la manière suivante :

- $f^{\prime}$ de classe $C^{1}$ par morceaux ;

- $\operatorname{div}_{z} \underline{f}^{\prime}=0,\left[\underline{f}^{\prime}\right] \cdot \underline{n}=0 \quad(\Sigma)$ où $\Sigma$ désigne une surface de discontinuité éventuelle du champ $\underline{f}^{\prime}$;

$-\underline{f}^{\prime} \cdot \underline{n}=D^{\gamma} \underline{H} \cdot \underline{n}\left(I^{f_{s}}\right)$

- $\left(\underline{f}^{\prime} \cdot \underline{n}\right) \underline{n}$ périodique.

On introduit sur $\boldsymbol{F}$ la fonctionnelle suivante :

$$
W^{*}\left(\underline{f}^{\prime}\right)=\frac{1}{2 D^{\gamma}} \int_{C^{f}} \underline{f}^{\prime 2} \mathrm{~d} V_{z}
$$

On démontre (annexe 2 : Principe du minimum en flux) que la solution $\underline{f}$ réalise le minimum de $W^{*}$ dans $F$ :

$$
\left(\forall \underline{f}^{\prime} \in \boldsymbol{F}\right) W^{*}(\underline{f}) \leq W^{*}\left(\underline{f^{\prime}}\right)
$$

D'une manière duale, on définit l'ensemble $\boldsymbol{R}$ des concentrations $r^{\prime}$ admissibles :

$$
\boldsymbol{R}=\left\{r^{\prime} C^{0} ; C^{1} / \text { morceaux }, \text { périodiques }\right\}
$$

et l'on définit sur $\boldsymbol{R}$ la fonctionnelle $W$ :

$$
\begin{aligned}
W\left(r^{\prime}\right)= & \frac{D^{\gamma}}{2} \int_{C^{f}} \underline{\operatorname{grad}} z r^{\prime 2} \mathrm{~d} V_{z} \\
& +D^{\gamma} \int_{I^{f s}} r^{\prime} \underline{H} \cdot \underline{n} \mathrm{~d} S_{z}
\end{aligned}
$$

On démontre (annexe 2 : Principe de minimum en concentration) que la solution $r$ réalise dans $\boldsymbol{R}$ le minimum de $W$ :

$$
\left(\forall r^{\prime} \in \boldsymbol{R}\right) W(r) \leq W\left(r^{\prime}\right)
$$

On montre encore que les minima de $W$ et $W^{*}$ réalisés par $r$ et $\underline{f}=-D^{\gamma} \underline{\operatorname{grad}}_{z} r$ respectivement sont opposés :

$$
W(r)+W^{*}(\underline{f})=0
$$

Enfin, en intégrant par parties l'expression de $W^{*}(\underline{f})$, et en utilisant les propriétés de périodicité de $r$ et $f$, on obtient :

$$
\begin{aligned}
W^{*}(\underline{f}) & =-\frac{1}{2} \int_{C^{f}} \underline{f} \cdot \underline{\operatorname{grad}} z \mathrm{~d} V_{z} \\
& =-\frac{D^{\gamma}}{2} \int_{I^{f s}} r \underline{H} \cdot \underline{n} \mathrm{~d} S_{z} \\
& =-\frac{D^{\gamma}}{2} \int_{C^{f}} \underline{H} \cdot \underline{\operatorname{grad}}_{z} r \mathrm{~d} V_{z}
\end{aligned}
$$

Compte tenu des équations (66) et (75), on déduit de l'équation (76) que :

$$
\frac{2 W(r)}{D^{\gamma}\left|C^{f}\right|}=-\frac{2 W^{*}(\underline{f})}{D^{\gamma}\left|C^{f}\right|}=\underline{H} \cdot{ }^{\mathrm{t}} \underline{\underline{\operatorname{grad}}}_{z}^{\underline{\chi}}{ }^{f} \cdot \underline{H}
$$

En combinant les équations (71), (74) et (77), on voit que la conjonction des deux principes variationnels conduit à un encadrement de la tortuosité :

$$
\begin{aligned}
\left(\forall \underline{f}^{\prime} \in \boldsymbol{F}\right)\left(\forall r^{\prime} \in \boldsymbol{R}\right) & -\frac{2 W^{*}\left(\underline{f^{\prime}}\right)}{D^{\gamma}\left|C^{f}\right|} \\
& \leq \underline{H} \cdot\left(\underline{\underline{T}}_{\mathrm{per}}-\underline{\underline{1}}\right) \cdot \underline{H} \leq \frac{2 W\left(r^{\prime}\right)}{D^{\gamma}\left|C^{f}\right|}
\end{aligned}
$$

ou encore, en termes de tenseur de diffusion :

$$
\begin{aligned}
\left(\forall \underline{f}^{\prime} \in \boldsymbol{F}\right)\left(\forall r^{\prime}\right. & \in \boldsymbol{R})-\frac{2 W^{*}\left(\underline{f}^{\prime}\right)}{D^{\gamma}|C|} \\
& \leq \underline{H} \cdot\left(\underline{\underline{D}}_{\mathrm{per}}^{\text {hom }}-\varphi^{\mathrm{f}} D^{\gamma} \underline{\underline{ }}\right) \cdot \underline{H} \leq \frac{2 W\left(r^{\prime}\right)}{D^{\gamma}|C|}
\end{aligned}
$$

Notons comme corrolaire immédiat de l'équation (78) l'inégalité $\underline{\underline{T}}_{\text {per }} \leq \underline{\underline{1}}$, au sens où :

$$
\forall \underline{H}, \underline{H} \cdot(\underline{\underline{T}} \text { per }-\underline{1}) \cdot \underline{H} \leq 0
$$

qui résulte du choix particulier $r^{\prime}=0$.

\subsection{Interprétation géométrique du coefficient de tortuosité}

Dans l'analyse physique du transport diffusif, on distingue classiquement trois effets majeurs :

- celui du volume disponible, mesuré par la porosité du matériau considéré ;

- celui de l'écart entre le chemin idéal et le chemin réellement emprunté par l'espèce diffusante à l'intérieur de la porosité connectée. Il s'agit du caractère tortueux du chemin à parcourir, qui justifie la terminologie de tortuosité;

- celui des effets de constriction, c'est-à-dire la résistance au transport introduite par la variation de la section transversale des tubes de diffusion.

\subsubsection{Origine classique de la tortuosité}

Dans cette section, on s'intéresse à l'effet du caractère tortueux du chemin à parcourir sur la valeur de la tortuosité.

Afin d'illustrer l'emploi de l'équation (78) et d'établir la cohérence entre l'approche micro-macro du concept de tortuosité et l'approche classique, on considère le cas particulier bidimensionnel où l'espace poreux est constitué de tronçons 
rectilignes. Pour simplifier l'exposé, on suppose que ces derniers sont d'épaisseur $\delta$ constante et alternativement horizontaux ou verticaux. La cellule de base est présentée en figure 7. On soumet le milieu poreux à un gradient de concentration macroscopique $\underline{H}=H \underline{e}_{x}$. Pour une longueur $L$ comptée parallèlement à $\underline{e}_{x}$ le trajet réel des particules est de l'ordre de $D=\Sigma_{i} d_{i}$. On rend compte classiquement d'une telle morphologie en corrigeant le coefficient de diffusion $D^{\gamma}$ par une tortuosité $(L / D)^{2}$ (Dullien, 1992). On se propose de montrer que la conjonction des approches variationnelles exposées à la section 4.2 permet d'obtenir très simplement un encadrement de la valeur exacte $T$ du coefficient de tortuosité, et que l'estimation classique $(L / D)^{2}$ est en fait un minorant de $T$.

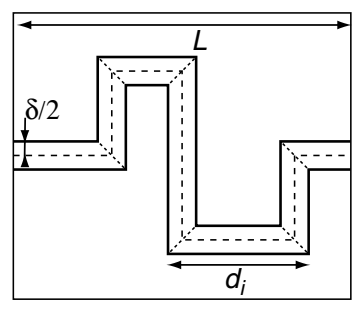

Approche en flux

Figure 7

Tronçons rectilignes perpendiculaires.

Perpendicular rectilinear section.

\section{Approche en flux}

On sépare deux tronçons successifs par une interface parallèle à l'une ou l'autre des bissectrices du dièdre $\left(\underline{e}_{x}, \underline{e}_{y}\right)$ (fig. 7). On considère alors un flux $j^{\gamma}$ dans la porosité égal à $J \underline{e}_{x}$ dans les tronçons horizontaux et à $J \underline{e}_{y}$ dans les tronçons verticaux, où $J$ est à ce stade un paramètre arbitraire. Le champ $f v^{\prime}=D^{\gamma} H+j^{\gamma^{\prime}}$ est manifestement admissible au sens de la section 4.2 et l'on vérifie que :

$$
\begin{aligned}
W^{*}\left(\underline{f v^{\prime}}\right)= & \frac{\delta}{2 D^{\gamma}}\left(\sum_{i \in I_{x}} d_{i}\left(D^{\gamma} H+J\right)^{2}\right. \\
& \left.+\sum_{i \in I_{y}} d_{i}\left(D^{\gamma 2} H^{2}+J^{2}\right)\right)
\end{aligned}
$$

où $I_{\alpha},(\alpha=x, y)$ désigne l'ensembles des indices $i$ correspondant à des tronçons parallèles à $\underline{e}_{\alpha}$. D'après l'équation (78), le choix optimal $J^{\text {opt }}$ de $J$ est obtenu en minimisant l'expression de $W^{*}\left(\underline{f v^{\prime}}\right)$ donnée par l'équation (80):

$$
\frac{\mathrm{d} W^{*}}{\mathrm{~d} J}\left(\underline{f v^{\prime}}\right)=0 \rightarrow J^{\mathrm{opt}}=-D^{\gamma} H \frac{L}{D}
$$

En reportant cette valeur dans l'équation (80), l'équation (78) conduit finalement à :

$$
\left(\frac{L}{D}\right)^{2} \leq T
$$

qui indique, comme annoncé, que l'estimation classique $(L / D)^{2}$ de la tortuosité est en fait un minorant de la valeur exacte.

\section{Approche en concentration}

On subdivise à présent la porosité en tronçons de longueur $\ell_{i}$ (fig. 7), séparés par des carrés de côté $\delta$ situés aux coudes du réseau. Étant donné un réel $A$, on introduit un champ de concentrations $\rho^{\gamma}$, dont la variation est affine en fonction de la coordonnée $\alpha$ dans les tronçons parallèles à $\underline{e}_{\alpha}(\alpha=x, y)$, soit $\rho^{\gamma}=A \alpha+B_{i}$, et qui est uniforme dans les carrés situés aux coudes du réseau. $A$ représente donc la norme du gradient de la concentration. On fixe $B_{1}=0$, les autres constantes $B_{i}$ étant choisies en sorte que $\rho^{\gamma}$, soit un champ continu.

On considère alors le champ $r^{\prime}=\rho^{\gamma}-H x$. L'unique valeur de $A$ assurant la périodicité de $r$ ' est :

$$
A=H \frac{L}{\ell} \quad \text { avec } \quad \ell=\sum_{i} \ell_{i}
$$

Pour ce choix de $A$, le champ $r^{\prime}$ est admissible $\left(r^{\prime} \in \boldsymbol{R}\right)$ et l'inégalité s'applique. Tous calculs faits, il vient :

$$
\begin{gathered}
W\left(r^{\prime}\right)=\frac{1}{2} D^{\gamma} \delta \ell H^{2}\left(\left(\frac{L}{\ell}\right)^{2}-1-2 X\right) \\
\text { avec } X=\frac{\Sigma_{i \in I_{y}} \delta}{\ell}
\end{gathered}
$$

Remarquant que $D=\ell(1+2 X)$, on trouve :

$$
\frac{2 W\left(r^{\prime}\right)}{D^{\gamma}\left|C^{f}\right| H^{2}}=\frac{(L / \ell)^{2}-1-2 X}{1+2 X}
$$

de sorte que l'équation (78) conduit à :

$$
T \leq(1+2 X)\left(\frac{L}{D}\right)^{2}
$$

En rapprochant les équations (86) et (82), on voit que :

$$
1 \leq \frac{T}{(L / D)^{2}} \leq 1+2 X
$$

L'équation (87) fait apparaître que l'estimation classique $(L / D)^{2}$ de la tortuosité est d'autant meilleure que l'épaisseur $\delta$ des tronçons est plus petite devant les longueurs $\ell_{i}$ de ces derniers, afin que $X<1$. Pour la morphologie considérée ici, l'approche variationnelle confirme donc que la correction à 
apporter au coefficient $D^{\gamma}$ pour décrire la diffusion dans un poreux provient de la différence entre le chemin en projection sur la direction du gradient macroscopique de concentration et le chemin « tortueux » auquel sont astreintes les molécules du fait de la présence du solide.

\subsection{2 Étude des effets de constriction}

On se propose ici d'analyser et de quantifier les effets de constriction dans le calcul de la tortuosité d'un milieu poreux. Pour ce faire, on considère un réseau poreux périodique bidimensionnel dont la cellule de base est représentée en figure 8. La porosité est constituée de canaux d'épaisseur alternativement égale à $e$ et $E$, et de longueur alternativement égale à $l$ et $L$. On cherche à étudier les variations de la tortuosité longitudinale en fonction des rapports $\varepsilon=e / E$ et $\lambda=l / L$.

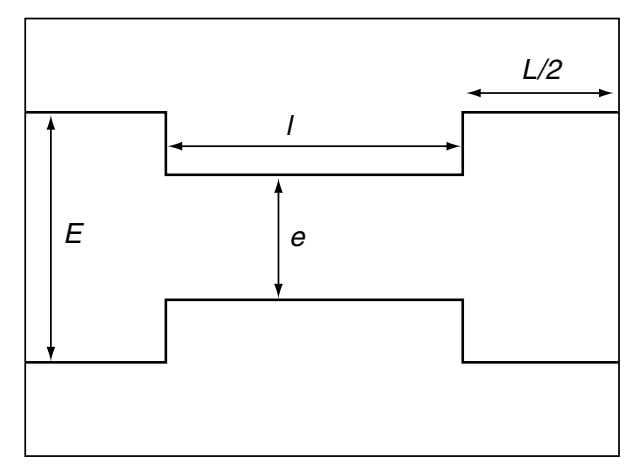

Figure 8

Cellule de base.

Elementary cell.

Approche en flux

On décompose le domaine effectivement occupé par le fluide saturant en plusieurs blocs dont la géométrie est paramétrée $\operatorname{par} \delta$ (fig. 9). Le flux $j^{\gamma^{\prime}}$ prend alors les valeurs suivantes :

$$
\underline{j^{\gamma^{\prime}}}= \begin{cases}\underline{V}=V \underline{e_{x}}, & \text { dans la grande section } \\ \underline{v}=v \underline{e_{x}}, & \text { dans la petite section } \\ \underline{0}, & \text { dans les coins } \\ w_{i}, & \text { ailleurs }\end{cases}
$$

Par ailleurs, les conditions d'interface entre les blocs permettent de réduire le nombre de paramètres du problème posé. La nullité de la discontinuité normale aux interfaces donne :

$$
\begin{aligned}
&\left(\underline{V}-\underline{w}_{i}\right) \cdot \underline{n}=0 \rightarrow V=w \cos \theta ; \\
& w=\left|w_{i}\right|(i=1,4) \\
&\left(\underline{v}-\underline{w}_{i}\right) \cdot \underline{n}=0 \rightarrow v=\frac{w \cos \theta}{\varepsilon}=\frac{V}{\varepsilon} ; \varepsilon=\frac{e}{E}
\end{aligned}
$$

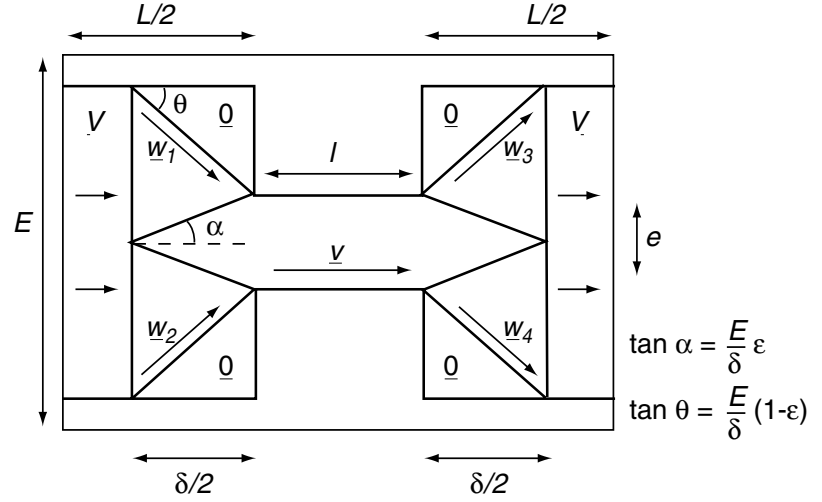

Figure 9

Approche en flux.

Variational approach on the diffusive flow field.

On se ramène donc à la résolution d'un problème à deux paramètres que l'on va chercher à optimiser. Le champ $\underline{f v^{\prime}}=D^{\gamma} \underline{H}+\underline{j^{\gamma^{\prime}}}$ est admissible au sens de la section 4.2 et l'on a :

$$
\begin{aligned}
W^{*}\left(\underline{f v^{\prime}}\right)= & \frac{\delta E}{4 D^{\gamma}}\left(D^{\gamma} H\right)^{2} \\
& +\frac{\delta E}{4 D^{\gamma}}\left[\left(D^{\gamma} H\right)^{2}+w^{2}+2 D^{\gamma} H w \cos \theta\right] \\
& +\frac{\varepsilon L E}{4 D^{\gamma}}\left(2 \lambda+\frac{\delta}{L}\right)\left[\left(D^{\gamma} H\right)^{2}+v^{2}+2 D^{\gamma} H v\right] \\
& +\frac{L E}{2 D^{\gamma}}\left(1-\frac{\delta}{L}\right)\left[\left(D^{\gamma} H\right)^{2}+V^{2}+2 D^{\gamma} H V\right]
\end{aligned}
$$

ou encore, en utilisant les résultats (89) et (90) et les relations géométriques présentées en figure 9 :

$$
\begin{aligned}
W^{*}\left(\underline{f v^{\prime}}\right) & =\frac{L E}{2 D^{\gamma}}\left((1+\lambda \varepsilon)\left(D^{\gamma} H\right)^{2}\right. \\
& \left.+\frac{\delta(1-\varepsilon)+L(\lambda+\varepsilon)}{L \varepsilon} V^{2}+2(\lambda+1) D^{\gamma} H V\right)
\end{aligned}
$$

D'après l'équation (78), le choix optimal $\left(\delta^{\text {opt }}, V^{\text {opt }}\right) \mathrm{du}$ couple $(\delta, V)$ est obtenu en minimisant l'expression de $W^{*}\left(\underline{f v^{\prime}}\right)$ donnée par l'équation (92) :

${\frac{\mathrm{d} W^{*}\left(\underline{f v^{\prime}}\right)}{\mathrm{d} \delta} \mid V, \text { fixe }}=0 \rightarrow \delta^{\mathrm{opt}}=E \sqrt{\varepsilon(1-\varepsilon)}$

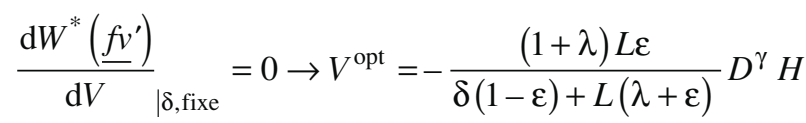


En reportant cette valeur dans l'équation (92) et en introduisant le paramètre adimensionnel $\Lambda=\delta / L$, l'équation (78) aboutit finalement à une estimation par défaut $T^{-}$de la tortuosité :

$$
\frac{\varepsilon[(1+\lambda \varepsilon)(1-\Lambda)+\lambda(1+\lambda)]}{(1+\lambda \varepsilon)[\Lambda(1-\varepsilon)+\lambda+\varepsilon]}=T^{-} \leq T
$$

où l'on vérifie $\lim _{\varepsilon \rightarrow 1} T^{-}=1$.

Le cas des fortes constrictions est celui où $\varepsilon<<1(\Lambda<<1)$. Dans ces conditions, si $\lambda=O(1)$, on voit que :

$$
T^{-} \sim \varepsilon\left(\lambda+1+\frac{1}{\lambda}\right)
$$

\section{Approche en concentration}

La porosité est à présent subdivisée en trois blocs d'épaisseurs respectives $E$, $e$ et $E$ (fig. 10). Soit $\rho^{\gamma}$, une fonction continue, affine par morceaux, avec $\mathrm{d} \rho^{\gamma^{\prime}} / \mathrm{d} x=H+\alpha$ (respectivement $\mathrm{d} \rho^{\gamma^{\prime}} / \mathrm{d} x=H+\beta$ ) dans les portions d'épaisseur $e$ (respectivement $E$ ) où $\alpha$ et $\beta$ sont des paramètres à définir. La continuité du champ $\rho^{\gamma}$ impose :

$$
\rho^{\gamma^{\prime}}= \begin{cases}(H+\beta) x & \text { dans le bloc } 1 \\ (H+\alpha) x+(\beta-\alpha) \frac{L}{2} & \text { dans le bloc } 2 \\ (H+\beta) x+(\alpha-\beta) \ell & \text { dans le bloc 3 }\end{cases}
$$

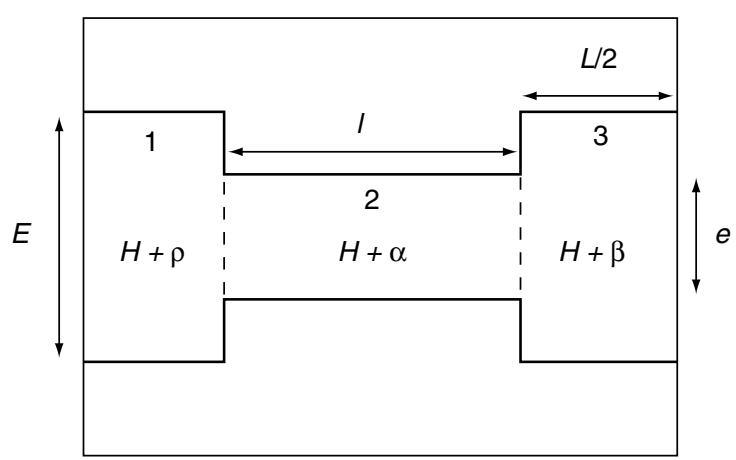

Figure 10

Approche en concentration.

Variational approach on the concentration field.

La condition de périodicité à laquelle le champ $r^{\prime}=\rho^{\gamma^{\prime}-} H x$ des fluctuations sur la cellule de base est astreint permet de définir une liaison entre $\alpha$ et $\beta$ :

$$
r^{\prime}(x=0)=r^{\prime}(x=\ell+L) \Leftrightarrow \beta=-\lambda \alpha
$$

Pour cette valeur de $\beta$, le champ $r^{\prime}$ est admissible $\left(r^{\prime} \in \boldsymbol{R}\right)$ et l'inégalité (78) s'applique. On a donc :

$$
W\left(r^{\prime}\right)=\frac{D^{\gamma} L E}{2} \alpha^{2} \lambda(\lambda+\varepsilon)-D^{\gamma} \lambda L E \alpha(1-\varepsilon) H
$$

On optimise le choix de $\alpha$ dans la relation (98) :

$$
\frac{\mathrm{d} W\left(r^{\prime}\right)}{\mathrm{d} \alpha}=0 \rightarrow \alpha^{\mathrm{opt}}=\frac{1-\varepsilon}{\lambda+\varepsilon} H
$$

et en reportant cette valeur dans l'équation (98), l'équation (78) donne :

$$
T \leq T^{+}=\frac{\varepsilon(1+\lambda)^{2}}{(1+\lambda \varepsilon)(\lambda+\varepsilon)}
$$

À nouveau, dans le cas des fortes constrictions, on observe que :

$$
T^{+} \sim \varepsilon\left(\lambda+2+\frac{1}{\lambda}\right)
$$

à rapprocher de l'équation (95).

Finalement, les résultats (94) et (100) fournissent un encadrement de la tortuosité due à la constriction en fonction des paramètres géométriques de la cellule de base :

$$
\frac{\varepsilon[(1+\lambda \varepsilon)(1-\Lambda)+\lambda(1+\lambda)]}{(1+\lambda \varepsilon)[\Lambda(1-\varepsilon)+\lambda+\varepsilon]} \leq T \leq \frac{\varepsilon(1+\lambda)^{2}}{(1+\lambda \varepsilon)(\lambda+\varepsilon)}
$$

qui se réduit dans le cas des fortes constrictions au résultat suivant :

$$
\varepsilon\left(\lambda+1+\frac{1}{\lambda}\right) \leq T \leq \varepsilon\left(\lambda+2+\frac{1}{\lambda}\right)
$$

\subsection{Estimations numériques du coefficient de diffusion dans le cas périodique}

Les estimations numériques du tenseur de diffusion relatives au cas périodique présentées dans cette section sont basées sur l'équation (77) et sur le principe de minimum en concentration qui conduit à l'inégalité de droite de l'équation (79). La minimisation de $W\left(r^{\prime}\right)$ est effectuée dans le cadre de la méthode des éléments finis. Pour un maillage donné, la solution du calcul en éléments finis qui réalise pour ce maillage le minimum de $W$ fournit une estimation par excès des tenseurs de diffusion et de tortuosité. Les maillages tridimensionnels ont été réalisés à l'aide d'éléments tétraédriques à 4 nœuds.

La figure 11 représente le coefficient diagonal du tenseur de diffusion $D_{\text {per }}^{\text {hom }}$ en fonction de la porosité. La cellule de base est cubique. Au centre de celle-ci, le solide occupe un domaine sphérique ou cubique (fig. 12). Dans le cas de l'inclusion sphérique, on notera que cette morphologie ne permet pas d'explorer des valeurs de la porosité inférieures à $1-\pi / 6$. Cette limitation disparaît pour l'inclusion de forme cubique. 


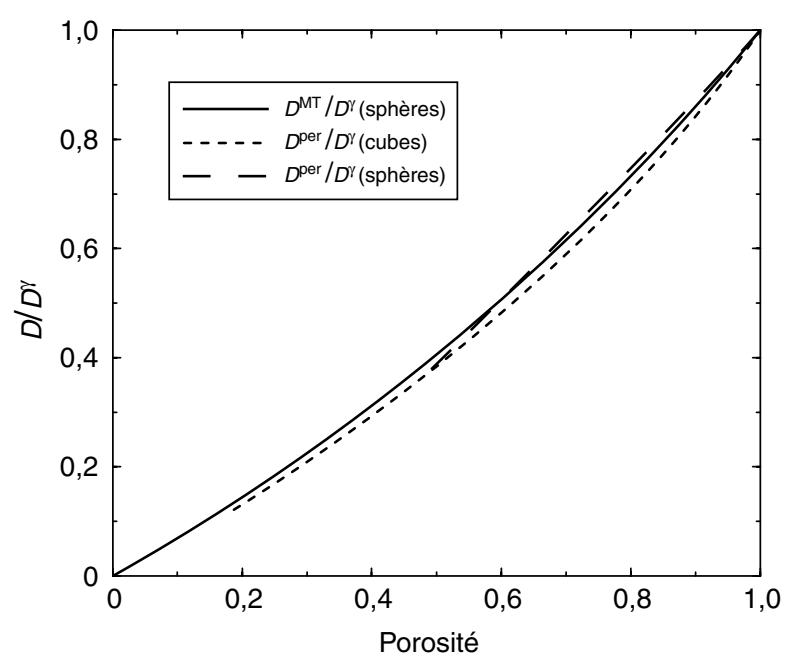

Figure 11

Cas périodique : inclusion solide sphérique ou cubique.

Periodic case: spherical or cubic solid inclusion.

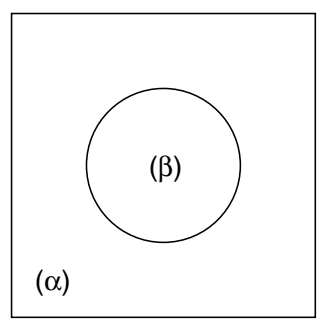

Inclusion sphérique

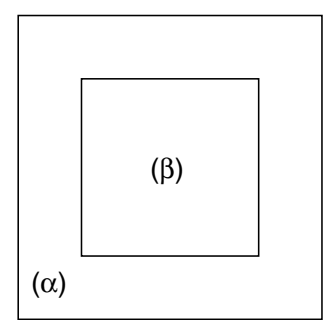

Inclusion cubique
Figure 12

Cellule de base cubique avec inclusion sphérique ou cubique.

Cubic elementary cell with spherical or cubic inclusion.

On constate que le schéma de Mori-Tanaka et l'approche périodique pour les deux cellules de base de la figure 12 fournissent des estimations très voisines. Par ailleurs, à porosité identique, on note que le passage de la forme sphérique à la forme cubique ne modifie pas significativement le résultat de l'approche périodique. L'intérêt de ces résultats réside dans le fait qu'ils justifient l'estimation analytique très simple de la formule (52).

La figure 13 compare les coefficients $D_{11}$ et $D_{33}$ du tenseur de diffusion prévus par le schéma de Mori-Tanaka pour un ellipsoïde $\varepsilon$ de rapport d'axes $a_{1} / a_{3}=10$ et les résultats de l'approche périodique. Le calcul périodique est effectué pour une cellule de base de forme parallélépipédique au centre de laquelle est placé un ellipsoïde de même rapport d'axes $a_{1} / a_{3}=10$ (fig. 14). $L_{i}$ désignant la demi-longueur de l'arète du parallélépipède colinéaire à la direction $\underline{e}_{i}$, on fixe le

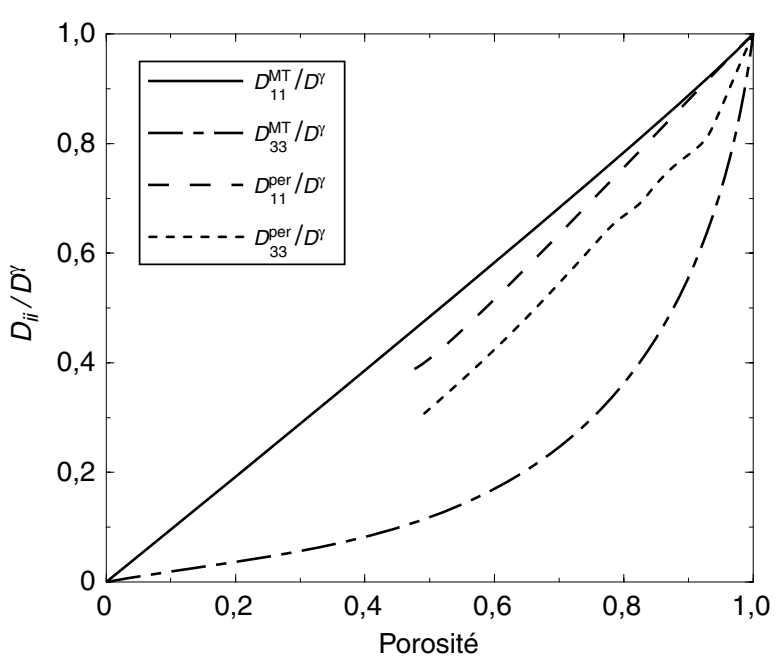

Figure 13

Cas périodique : inclusion solide ellipsoïdale, $a_{1} / a_{3}=10$.

Periodic case: ellipsoidal solid inclusion, $a_{1} / a_{3}=10$.
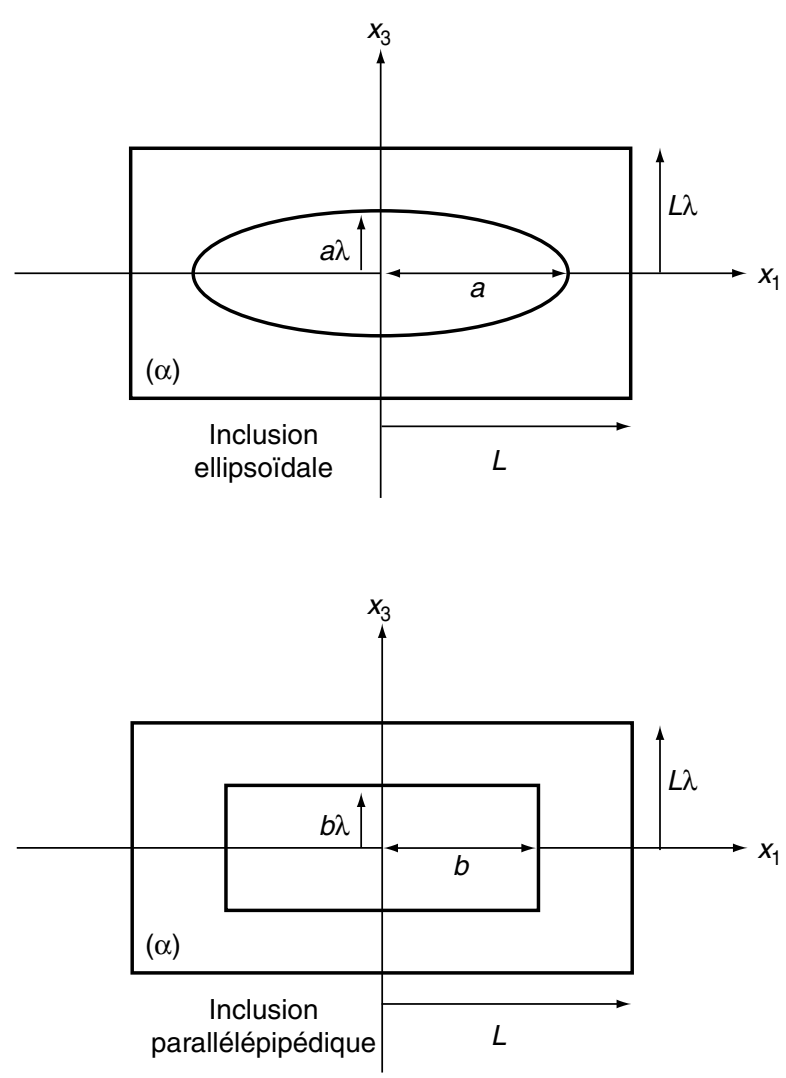

Figure 14

Cellule de base parallélépipédique avec inclusion ellipsoïdale ou parallélépipédique.

Parallelepipedic elementary cell with ellipsoidal or parallelepipedic inclusion. 
rapport $L_{1} / L_{3}$ égal à $a_{1} / a_{3}$, de sorte que la porosité du réseau périodique correspondant est indépendante de l'aplatissement de l'ellipsoïde, égale à $1-\frac{\pi}{6}\left(\frac{a_{1}}{L_{1}}\right)^{3}$. Pratiquement, les différentes valeurs de la porosité sont obtenues, sans modification du parallélépipède, en augmentant le volume de l'ellipsoïde de façon homothétique. Comme dans le cas d'inclusion sphérique, la valeur minimale accessible est $1-\pi / 6$. D'une part, contrairement ce que prévoit le schéma de MoriTanaka, on observe que l'aplatissement de l'ellipsoïde ne fait pas tendre le calcul périodique de $D_{11}$ vers la loi des mélanges (section 3.1). D'autre part, le rapport $D_{11} / D_{33}$ qui mesure l'anisotropie du transport diffusif est beaucoup plus faible dans le cas périodique que dans l'estimation de MoriTanaka. Ces deux constatations sont commentées successivement ci-après.

On s'intéresse tout d'abord à l'effet de l'aplatissement sur les coefficients $D_{11}$ et $T_{11}$.

Pour effectuer un calcul analytique simple, il est commode de remplacer l'inclusion solide ellipsoïdale par une inclusion de forme parallélépipédique, comme à la figure 14 . On note $b_{1}$ (respectivement $L_{1}$ ) la demi-longueur des arètes de l'inclusion (respectivement de la cellule de base) parallèles à $\underline{e}_{1}$ et l'on pose $X=b_{1} / L_{1}$. La morphologie particulière de l'inclusion placée au centre de la cellule de base invite à explorer des fluctuations $r$ impaires par rapport à $x_{1}$. On pose donc $r_{A}^{\prime}=A \sin \left(\pi x_{1} / L\right)$. En optimisant le choix du paramètre $A$, on montre que l'inégalité de droite de l'équation (78) conduit à :

$$
\begin{gathered}
T_{11}^{\text {per }} \leq 1-g(X) \\
g(X)=\frac{2 X^{4} \sin ^{2} \pi X}{\pi^{2}\left(1-X^{3}\right)\left(1-X^{3}\left(1+\frac{\sin 2 \pi X}{2 \pi X}\right)\right)}
\end{gathered}
$$

L'existence d'une majoration de $T_{11}^{\text {per }}$ indépendante de l'aplatissement quantifié par $\lambda$ confirme le résultat du calcul numérique selon lequel $T_{11}^{\text {per }}$ ne tend pas vers 1 . Il convient cependant de souligner le fait que la majoration (104) est tributaire de la morphologie particulière examinée ici, où l'inclusion est placée au centre de la cellule de base. En particulier, le fait qu'elle soit indépendante de l'aplatissement ne possède aucune généralité.

La deuxième constatation issue de la figure 13 porte sur le fait que les valeurs de $D_{33}^{\text {per }}$ sont significativement supérieures à celles de l'estimation $D_{33}^{\mathrm{MT}}$ de Mori-Tanaka. En d'autres termes, le calcul périodique fournit une valeur plus élevée du coefficient de tortuosité $T_{33}$. Cette remarque conduit à incriminer la trop grande simplicité de la morphologie de la figure 14. En effet, celle-ci se révèle insuffisamment «tortueuse » vis-à-vis d'un gradient macroscopique colinéaire à $\underline{e}_{3}$ au sens où, contrairement au schéma de la figure 6, l'aplatissement des particules solides n'interdit pas l'existence de trajets rectilignes colinéaires à $\underline{e}_{3}$. Ce point peut être précisé au moyen d'une approche variationnelle en flux. Soit donc $\boldsymbol{V}$ le cylindre dont les génératrices, colinéaires à $\underline{e}_{3}$, s'appuient sur le contour de l'ellipsoïde de la figure 14 . On considère le champ $j^{\gamma}$ égal à $-D^{\gamma} H \underline{e}_{3}$ en dehors de $\boldsymbol{V}$ et nul à l'intérieur de $\boldsymbol{V}$. En appliquant l'inégalité de gauche de l'équation (79) à la fluctuation $\underline{f}=\underline{j}^{\gamma^{\prime}}+D^{\gamma} H \underline{e}_{3}$, on obtient un minorant noté $D_{33}^{\min }$ de $D_{33}^{\text {per }}$ :

$\frac{D_{33}^{\text {per }}}{D^{\gamma}} \geq D_{33}^{\min }=1-\frac{\pi}{4}\left(\frac{a_{1}}{L_{1}}\right)^{2}=1-\frac{\pi}{4}\left(\frac{6}{\pi}\left(1-\varphi^{f}\right)\right)^{2 / 3}$

La figure 15 montre que les variations de $D_{33}^{\text {per }}$ et $D_{33}^{\min }$ en fonction de la porosité sont très voisines, toutes deux nettement au-dessus de $D_{33}^{\mathrm{MT}}$. Ce bon accord confirme que le champ $j^{\gamma}$ est une estimation satisfaisante du flux diffusif qui a donc lieu essentiellement en dehors du cylindre $\boldsymbol{V}$, sur des trajets faiblement tortueux.

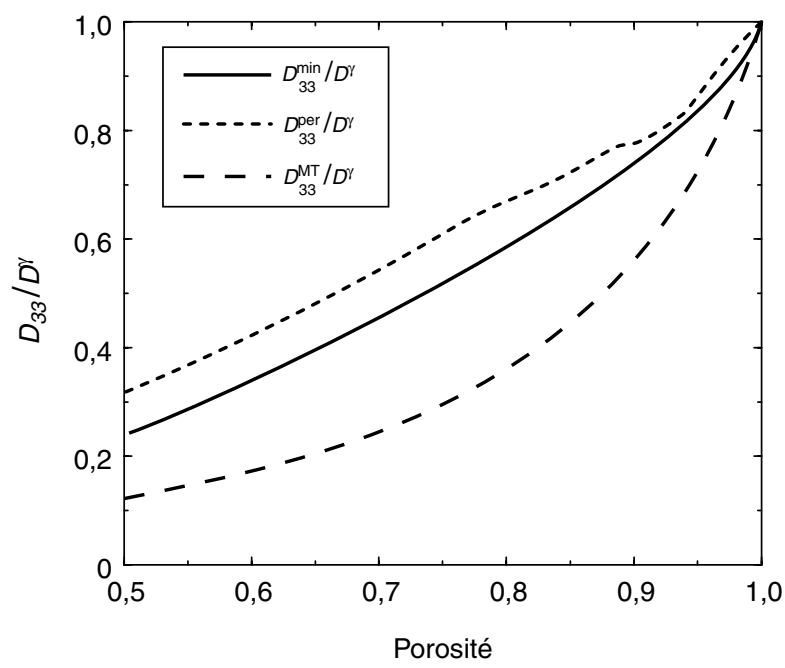

Figure 15

Variations de $D_{33}^{\text {per }}, D_{33}^{\mathrm{MT}}, D_{33}^{\mathrm{min}}$

Variations of $D_{33}^{\mathrm{per}}, D_{33}^{\mathrm{MT}}, D_{33}^{\mathrm{min}}$.

\section{CONCLUSION}

Pour une advection modérée $\left(V \leq D^{\gamma} / \boldsymbol{L}\right)$ et un régime transitoire suffisamment lent $\left(t_{c} \geq \boldsymbol{L}^{2} / D^{\gamma}\right)$, le transport diffusif est homogénéisable. Sous ces conditions et en négligeant la compressibilité de la phase fluide, le flux diffusif macroscopique d'un soluté est relié de façon linéaire au gradient macroscopique de sa masse volumique à l'aide d'un tenseur 
de diffusion, produit du coefficient de diffusion en solution infinie, de la porosité et d'un tenseur de tortuosité. Ce dernier, fonction des seuls paramètres morphologiques de l'échelle microscopique, peut être déterminé par une méthode de changement d'échelle d'espace à partir d'un problème purement diffusif posé à l'échelle microscopique.

Les méthodes d'estimation utilisées classiquement dans les problèmes de thermique (Hashin, 1968) ou d'élasticité des matériaux hétérogènes fournissent alors des expressions analytiques simples de la tortuosité dans le cas isotrope où le solide peut être modélisé par des inclusions sphériques. Elles permettent aisément d'analyser l'anisotropie du phénomène de diffusion quand la phase solide est décrite au moyen d'inclusions ellipsoïdales de forme et orientation identiques. Pour une inclusion aplatie possédant la symétrie de révolution autour de son petit axe, le coefficient de tortuosité dans les directions des grands axes tend vers 1 , tandis que l'anisotropie augmente avec le rapport des axes de l'ellipsoïde.

La méthode de l'homogénéisation périodique (Auriault et Lewandowska, 1996) fournit un cadre théorique rigoureux et fécond pour la recherche de la formulation des lois du transport à l'échelle macroscopique. Cependant, la détermination pratique du tenseur de diffusion macroscopique, qui repose sur la résolution numérique d'un problème elliptique à conditions aux limites périodiques, est évidemment plus lourde, même pour des morphologies simples. Dans le cas d'inclusions sphériques, l'accord avec les méthodes d'estimation est excellent. En revanche, il n'est pas possible de rendre compte de l'anisotropie du transport diffusif induit par l'aplatissement des particules de solides avec une morphologie simple, du type de celles de la figure 14 . Le recours à une cellule de base plus complexe devrait toutefois permettre de pallier cette insuffisance.

\section{RÉFÉRENCES}

Auriault, J.-L. et Lewandowska, Y. (1996) Diffusion/ Adsorption/Advection Macrotransport in Soils. Eur. J. Mech. A/Solids, 15, 4, 681-704.

Bear, J. et Bachmat, Y. (1990) Introduction to Modeling of Transport Phenomena in Porous Media, Kluwer Academic Publishers.

Carslaw, H.S. et Jaeger, J.C. (1959) Conduction of Heat in Solids, 2 éd., Clarendon Press.

Dullien, F.A.L. (1992) Porous Media, Fluid Transport and Pore Structure, Academic Press.

Eshelby, J.D. (1957) The Determination of the Elastic Field of an Ellipsoidal inclusion, and Related Problems. Proc. R. Soc. A, 349, 261, 376-396.

Eshelby, J.D. (1961) Elastic Inclusion and Inhomogeneities, in Progress in Solid Mechanics, vol. II, Sneddon, I.N. et Hill, R. (eds.), North-Holland Pub. Co., 87-140.

Gilbert, F. (1987) Descriptions thermo-mécaniques de milieux à plusieurs constituants et application aux milieux poreux saturés, Thèse, Paris VI.

Hashin, Z. (1968) Assessment of the Self Consistent Scheme Approximation: Conductivity of Particular Composites. J. Composite Materials, 2, 3, 284-300.

Hashin, Z. et Shtrikman, S. (1963) A Variational Approach to the Theory of the Elastic Behaviour of Multiphase Materials. J. Mech. Phys. Solids, 11, 127-140.

Maxwell, J.C. (1892) A Treatise on Electricity and Magnetism, 2 vol., Clarendon Press.

Willis, J.R. (1977) Bounds and Self-Consistent Estimates for the Overall Properties of Anisotropic Composites. J. Mech. Phys. Solids, 25, 185-202.

Zaoui, A. (1996) Matériaux hétérogènes et composites. Cours de l'École polytechnique.

Zaoui, A. (1997) Structural Morphology and Constitutive Behaviour of Microheterogeneous Materials, in Continuum Micromechanics, CISM Courses and Lectures No. 377, Suquet, P. (ed.), Springer.

Manuscrit définitif reçu en mai 1999 


\section{ANNEXE 1}

Dans le cadre du schéma différentiel (section 3.2), la mise en œuvre du schéma dilué à chaque itération nécessite de connaître les tenseurs $\stackrel{R}{=}_{F}^{\varepsilon k}$ de l'équation (55). Ceux-ci proviennent de la résolution du problème de l'inhomogénéité ellipsoïdale dans un milieu infini qui est ici anisotrope, les directions principales du tenseur de diffusion coïncidant avec les axes de l'ellipsoïde. On se limite dans la suite au cas où $\varepsilon$ possède la symétrie de révolution autour de l'axe dirigé par le vecteur $\underline{e}_{3}$ et où le milieu infini est isotrope transverse par rapport à cette direction. Le tenseur $\underline{R}_{\gamma}^{\varepsilon}$ intervenant dans l'estimation de Mori-Tanaka (49) correspond au cas particulier où le milieu infini est isotrope.

L'approche d'Eshelby (1957) consiste à examiner tout d'abord un problème auxiliaire dit de l'inclusion, dont on déduit la solution du problème de l'inhomogénéité. La dernière section traite le cas particulier de l'estimation de MoriTanaka.

\section{Inclusion ellipsoïdale dans un milieu isotrope transverse}

On considère un domaine infini homogène de coefficient de diffusion isotrope transverse $\underline{\underline{D}}_{0}$ :

$$
\underline{\underline{D}}_{0}=D_{h}\left(\underline{e}_{1} \otimes \underline{e}_{1}+\underline{e}_{2} \otimes \underline{e}_{2}\right)+D_{v} \underline{e}_{3} \otimes \underline{e}_{3}
$$

où $\left(\underline{e}_{1}, \underline{e}_{2}, \underline{e}_{3}\right)$ est un repère orthonormé de l'espace. Soit $\varepsilon$ un ellipsoïde centré à l'origine dont les axes sont colinéaires aux $\underline{e}_{i}$, de demi-longueurs $a_{i}$, avec $a_{1}=a_{2}$. Étant donné un vecteur $\underline{p}^{\varepsilon}$, on s'intéresse au problème suivant, dit de l'inclusion :

$$
\operatorname{div} \underline{j}=0 ; \underline{j}=-\underline{\underline{D}}_{0} \cdot \underline{\operatorname{grad}} \rho+\underline{p}^{\varepsilon} \chi^{\varepsilon}
$$

avec la condition à l'infini :

$$
\rho \rightarrow 0 \text { quand }|\underline{z}| \rightarrow \infty
$$

où $\chi^{\varepsilon}$ est la fonction caractéristique de l'ellipsoïde $\varepsilon$. Notant $\Delta^{\eta}$ l'opérateur :

$$
\Delta^{n}=\frac{\partial}{\partial z_{1}^{2}}+\frac{\partial}{\partial z_{2}^{2}}+\eta \frac{\partial}{\partial z_{3}^{2}} \text { avec } \eta=\frac{D_{v}}{D_{h}}
$$

et $\delta_{\partial \varepsilon}$ la distribution de Dirac dont le support est la frontière $\partial \varepsilon$ de l'ellipsoïde, il s'agit de déterminer la solution du problème défini par l'équation (3) et :

$$
\Delta^{n} \rho=-\frac{1}{D_{h}} \underline{p}^{\varepsilon} \cdot \underline{n} \delta_{\partial \varepsilon}
$$

La solution $\rho_{0}$ du problème élémentaire défini par l'équation (3) et $\Delta^{\eta} \rho=\delta_{0}$ est donnée par :

$$
\rho_{0}\left(z_{1}, z_{2}, z_{3}\right)=-\frac{1}{4 \pi \sqrt{\eta} \sqrt{z_{1}^{2}+z_{2}^{2}+z_{3}^{2} / \eta}}
$$

On obtient la solution de (3)-(5) à partir de la solution élémentaire $\rho_{0}$ par intégration sur la frontière de l'ellipsoïde :

$$
\begin{aligned}
& \rho\left(z_{1}, z_{2}, z_{3}\right)=-\frac{1}{D_{h}} \underline{p}^{\varepsilon} \\
& \cdot \int_{\partial \varepsilon} \underline{n}\left(Z_{1}, Z_{2}, Z_{3}\right) \rho_{0}\left(z_{1}-Z_{1}, z_{2}-Z_{2}, z_{3}-Z_{3}\right) \mathrm{d} S_{Z_{1}, Z_{2}, Z_{3}}
\end{aligned}
$$

Soit $\bar{\varepsilon}$ l'ellipsoïde image de $\varepsilon$ par l'application $\overline{Z_{1}}=Z_{1}$; $\overline{Z_{2}}=Z_{2} ; \overline{Z_{3}}=Z_{3} / \sqrt{\eta}$. En transformant l'équation (7) en intégrale de volume sur $\bar{\varepsilon}$, on obtient :

$$
\rho\left(z_{1}, z_{2}, z_{3}\right)=-\frac{1}{4 \pi D_{h}} p_{j}^{\varepsilon} \frac{\partial}{\partial z_{j}}\left(\varnothing\left(z_{1}, z_{2}, z_{3} / \sqrt{\eta}\right)\right)
$$

où $\emptyset$ est le potentiel de l'ellipsoïde $\bar{\varepsilon}$ :

$$
\varnothing\left(z_{1}, z_{2}, z_{3} / \sqrt{\eta}\right)
$$

$$
=\int_{\bar{\varepsilon}} \frac{\mathrm{d} Z_{1}, \mathrm{~d} Z_{2}, \mathrm{~d} Z_{3}}{\sqrt{\left(z_{1}-Z_{1}\right)^{2}+\left(z_{2}-Z_{2}\right)^{2}+\left(z_{3} / \sqrt{\eta}-Z_{3}\right)^{2}}}
$$

Finalement, en dérivant l'équation (8), on obtient une expression de $\operatorname{grad} \rho$ dans $\varepsilon$ sous la forme suivante :

$$
\underline{\operatorname{grad}} \rho\left(z_{1}, z_{2}, z_{3}\right)=\underline{\underline{R}}{ }_{0}^{\varepsilon} \cdot p^{\varepsilon}
$$

$$
\text { avec } R_{0}^{\varepsilon} i j=-\frac{1}{4 \pi D_{h}} \frac{\partial^{2}}{\partial z_{i} z_{j}}\left(\varnothing\left(z_{1}, z_{2}, z_{3} / \sqrt{\eta}\right)\right)
$$

où l'indice 0 dans $\underline{R}_{0}^{\varepsilon}$ rappelle que le résultat est relatif au milieu infini de coefficient de diffusion $\underline{\underline{D}}_{0}$. Compte tenu de l'équation (41) (section 3.1), la matrice de $\underline{R}_{0}^{\varepsilon}$ est diagonale dans la base $\left(\underline{e}_{1}, \underline{e}_{2}, \underline{e}_{3}\right)$ :

$$
R_{011}^{\varepsilon}=\frac{I_{1}(\bar{\varepsilon})}{4 \pi D_{h}} ; R_{022}^{\varepsilon}=R_{011}^{\varepsilon} ; R_{033}^{\varepsilon}=\frac{I_{3}(\bar{\varepsilon})}{4 \pi D_{v}}
$$


Dans le cas d'un ellipsoïde $\varepsilon$ de révolution «aplati », c'est-à-dire satisfaisant $a_{1}=a_{2}>a_{3}$, on pose $w=a_{3} / a_{1}$. Rappelons que :

$$
\begin{gathered}
I_{1}(\varepsilon)=I_{2}(\varepsilon)=\frac{2 \pi w}{\left(1-w^{2}\right)^{3 / 2}}\left(\arccos (w)-w\left(1-w^{2}\right)^{1 / 2}\right) ; \\
I_{3}(\varepsilon)=4 \pi-2 I_{1}(\varepsilon)
\end{gathered}
$$

On introduira donc dans l'équation (11) les valeurs suivantes :

$$
\begin{aligned}
& I_{1}(\bar{\varepsilon})=I_{2}(\bar{\varepsilon}) \\
& =\frac{2 \pi w}{\sqrt{\eta}\left(1-\frac{w^{2}}{\eta}\right)^{3 / 2}}\left(\arccos \left(\frac{w}{\sqrt{\eta}}\right)-\frac{w}{\sqrt{\eta}}\left(1-\frac{w^{2}}{\sqrt{\eta}}\right)^{1 / 2}\right) \\
& I_{3}(\bar{\varepsilon})=4 \pi-2 I_{1}(\bar{\varepsilon})
\end{aligned}
$$

Compte tenu de l'équation (10), le gradient dans $\varepsilon$ de la solution de l'équation (2) et satisfaisant :

$$
\rho \rightarrow \underline{H} \cdot \underline{z} \quad \text { quand } \quad|\underline{z}| \rightarrow \infty
$$

est uniforme et donné par :

$$
\underline{\operatorname{grad}} \rho\left(z_{1}, z_{2}, z_{3}\right)=\underline{\underline{R}}_{0}^{\varepsilon} \cdot \underline{p}^{\varepsilon}+\underline{H}
$$

\section{Inhomogénéité ellipsoïdale dans un milieu isotrope transverse}

On place une inhomogénéité ellipsoïdale $\varepsilon$ de coefficient de diffusion $D^{s} \underline{\underline{1}}$ dans un milieu infini $\Omega$ de coefficient de diffusion $\underline{\underline{D}}_{0}$. Les équations du problème de l'inhomogénéité sont :

$$
\begin{aligned}
& \operatorname{div} \underline{j}=0 \\
& \underline{j}=-\underline{\underline{D}}(\underline{x}) \cdot \underline{\operatorname{grad} \rho} \quad \text { avec } \quad\left\{\begin{array}{l}
\underline{D}(\underline{x})=D^{s} \underline{1} \text { dans } \varepsilon \\
\underline{\underline{D}}(\underline{x})=\underline{D}_{0} \text { dans } \Omega-\varepsilon
\end{array}\right.
\end{aligned}
$$

On complète l'équation (16) par la condition (14). On observe que l'équation (16) s'écrit formellement comme l'équation (2), quitte à poser :

$$
\underline{p}^{\varepsilon}=-\delta \underline{\underline{D}} \cdot \underline{\operatorname{grad}} \rho \quad \text { avec } \quad \delta \underline{\underline{D}}=-D^{s} \underline{\underline{1}}-\underline{\underline{D}}_{0}
$$

En rapprochant les équation (15) et (17), on voit que la valeur (uniforme dans $\varepsilon$ ) de $\operatorname{grad} \rho$ pour le problème de l'inhomogénéité est donnée en fonction de $\underline{H}$ par :

$$
(\underline{\operatorname{grad}} \rho)^{\varepsilon}=\left(\underline{\underline{1}}+\underline{\underline{R}}_{0}^{\varepsilon} \cdot \delta \underline{\underline{D}}\right)^{-1} \cdot \underline{H}
$$

\section{Estimation de Mori-Tanaka dans le cas ellipsoïdal de révolution}

Pour un ellipsoïde aplati de révolution autour de l'axe $\underline{e}_{3}\left(a_{1}=a_{2}>a_{3}\right)$, l'estimation de Mori-Tanaka conduit à un milieu homogénéisé isotrope transverse. Puisque le milieu de référence est isotrope $\left(\underline{\underline{D}}_{0}=D^{\gamma} \underline{\underline{1}}\right)$, le tenseur $\underline{\underline{R}}_{\gamma}^{\varepsilon}$ de l'équation (39) (section 3.1) est calculé à l'aide de l'équation (11) en faisant $\bar{\varepsilon}=\varepsilon$ et $D_{h}=D_{v}=D^{\gamma}$. Compte tenu de l'équation (49) (section 3.1), on obtient :

$$
T_{i i}^{\mathrm{MT}}=\frac{1-\frac{I_{i}(\varepsilon)}{4 \pi}}{\left(1-\varphi^{f}\right)+\varphi^{f}\left(1-\frac{I_{i}(\varepsilon)}{4 \pi}\right)}(\text { sans sommation sur } i)
$$

où les $I_{i}(\varepsilon)$ sont donnés par l'équation (12).

\section{ANNEXE 2}

Comme dans la section 4.2, on note $r$ et $\underline{f}=-D^{\gamma} \underline{\operatorname{grad}}_{z} r$ les champs du couple solution du problème direct (61)(63)-(64)-(65).

\section{Principe du minimum en flux}

Étant donné un élément $\underline{f}^{\prime}$ de $\boldsymbol{F}$, on pose $\underline{f}^{\prime}=\underline{f}+\delta \underline{f}$. Le champ $\delta \underline{f}$ possède les propriétés des flux admissibles, à l'exception de la condition de flux à l'interface $I^{f s}$ où l'on a $\delta \underline{f} \cdot \underline{n}=0$. En vertu de la définition (70) de la fonctionnelle $W^{*}$ (section 4.2), on voit que :

$$
W^{*}\left(\underline{f^{\prime}}\right)=W^{*}(\underline{f})+W^{*}(\delta \underline{f})+\frac{1}{D^{\gamma}} \int_{C^{f}} \underline{f} \cdot \delta \underline{f} \mathrm{~d} V_{z}
$$

En utilisant le fait que $\underline{f}=-D^{\gamma} \underline{\operatorname{grad}}_{z} r$, l'intégrale dans le membre de droite de l'équation (1) s'écrit en fonction de $r$ :

$$
\frac{1}{D^{\gamma}} \int_{C^{f}} \underline{f} \cdot \delta \underline{f} \mathrm{~d} V_{z}=\int_{C^{f}} r \operatorname{div} \delta \underline{f} \mathrm{~d} V_{z}-\int_{\partial C^{f}} r \delta \underline{f} \cdot \underline{n} \mathrm{~d} S_{z}
$$

Dans le membre de droite de l'équation (2), l'intégrale de volume est nulle en raison de la condition $\operatorname{div}_{z} \delta \underline{f}=0$. L'intégrale de surface est nulle en raison de la condition $\delta f \underline{n}=0$ sur $I^{f s}$ et des propriétés de périodicité de $r$ et $\delta f$ à la frontière de la cellule de base. L'équation (71) (section 4.2) résulte alors de la positivité de $\mathrm{W}^{*}(\delta f)$. 


\section{Principe de minimum en concentration}

Pour un élément $r^{\prime}$ de $\boldsymbol{R}$, on pose de même $r^{\prime}=r+\delta r$, en observant que $\delta r \in \boldsymbol{R}$. Compte tenu de l'équation (64) (section 4.2), on remarque d'abord que :

$$
\int_{C^{f}} \underline{\operatorname{grad}}_{z} \delta r \cdot \underline{\operatorname{grad}}_{z} r \mathrm{~d} V_{z}=\int_{\partial C^{f}} \delta r \underline{\operatorname{grad}} z \cdot \underline{n} \mathrm{~d} S_{z}
$$

En utilisant l'équation (65) (section 4.1) et les propriétés de périodicité de $\delta r$ et $\partial r / \partial n$ de l'équation (3), on obtient :

$$
\int_{C^{f}}^{\operatorname{grad}} z \delta \cdot \underline{\operatorname{grad}} z r \mathrm{~d} V_{z}+\int_{I^{f_{S}}} \delta \underline{H} \cdot \underline{n} \mathrm{~d} S_{z}=0
$$

On déduit des équations (73) (section 4.2) et (4) que :

$$
W\left(r^{\prime}\right)=W(r)+\frac{D^{\gamma}}{2} \int_{C^{f}}(\underline{\operatorname{grad}} z r)^{2} \mathrm{~d} V_{z}
$$

qui prouve l'inégalité (74) (section 4.2). 